\title{
Ion channel profiling of the Lymnaea stagnalis ganglia via transcriptome analysis
}

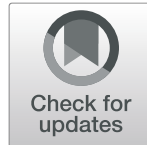

Nancy Dong ${ }^{1 \dagger}$, Julia Bandura ${ }^{1 \dagger}$, Zhaolei Zhang ${ }^{2}$, Yan Wang ${ }^{3,4}$, Karine Labadie $^{5}$, Benjamin Noel $^{6}$, Angus Davison ${ }^{7}$, Joris M. Koene ${ }^{8}$, Hong-Shuo Sun ${ }^{1,9}$, Marie-Agnès Coutellec ${ }^{10}$ and Zhong-Ping Feng ${ }^{1 *}$

\begin{abstract}
Background: The pond snail Lymnaea stagnalis (L. stagnalis) has been widely used as a model organism in neurobiology, ecotoxicology, and parasitology due to the relative simplicity of its central nervous system (CNS). However, its usefulness is restricted by a limited availability of transcriptome data. While sequence information for the L. stagnalis CNS transcripts has been obtained from EST libraries and a de novo RNA-seq assembly, the quality of these assemblies is limited by a combination of low coverage of EST libraries, the fragmented nature of de novo assemblies, and lack of reference genome.
\end{abstract}

Results: In this study, taking advantage of the recent availability of a preliminary L. stagnalis genome, we generated an RNA-seq library from the adult L. stagnalis CNS, using a combination of genome-guided and de novo assembly programs to identify 17,832 protein-coding L. stagnalis transcripts. We combined our library with existing resources to produce a transcript set with greater sequence length, completeness, and diversity than previously available ones. Using our assembly and functional domain analysis, we profiled L. stagnalis CNS transcripts encoding ion channels and ionotropic receptors, which are key proteins for CNS function, and compared their sequences to other vertebrate and invertebrate model organisms. Interestingly, L. stagnalis transcripts encoding numerous putative $\mathrm{Ca}^{2+}$ channels showed the most sequence similarity to those of Mus musculus, Danio rerio, Xenopus tropicalis, Drosophila melanogaster, and Caenorhabditis elegans, suggesting that many calcium channel-related signaling pathways may be evolutionarily conserved.

Conclusions: Our study provides the most thorough characterization to date of the L. stagnalis transcriptome and provides insights into differences between vertebrates and invertebrates in CNS transcript diversity, according to function and protein class. Furthermore, this study provides a complete characterization of the ion channels of Lymnaea stagnalis, opening new avenues for future research on fundamental neurobiological processes in this model system.

Keywords: Lymnaea stagnalis, Ion channels, Ionotropic receptors, Transcriptome, de novo assembly, CNS

\footnotetext{
*Correspondence: zp.feng@utoronto.ca

${ }^{\dagger}$ Nancy Dong and Julia Bandura contributed equally to this work.

'Department of Physiology, University of Toronto, 3308 MSB, 1 King's College

Circle, Toronto, ON M5S 1A8, Canada

Full list of author information is available at the end of the article
}

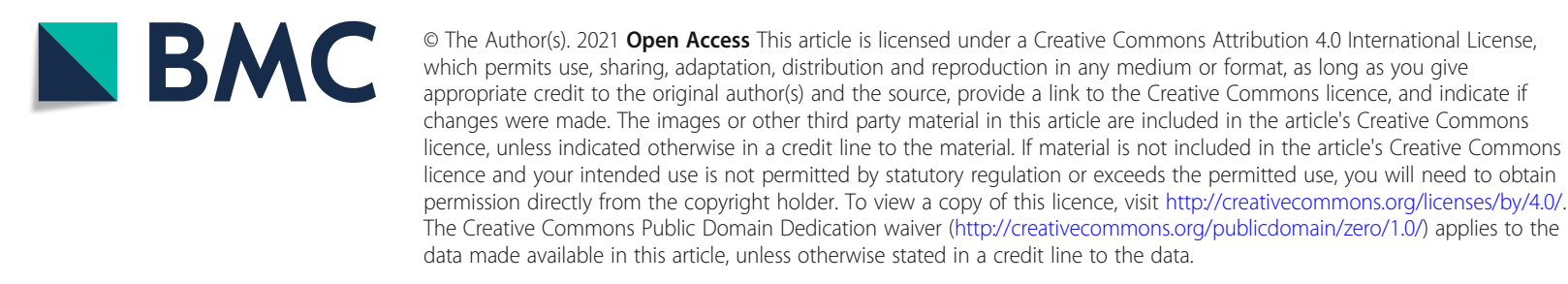




\section{Background}

The pond snail Lymnaea stagnalis is a widely used model organism in neurobiology, development, ecotoxicology, and parasitology. Its central nervous system (CNS) consists of large singly identifiable neurons and simple, well-characterized neural circuits, which allows detailed electrophysiological, biochemical, and molecular analyses of the cellular basis of behavior, including sensorimotor integration [1], learning and memory [2, 3], central pattern generator (CPG) networks [4], and neuromodulation [5]. In addition, L. stagnalis also exhibits notable traits absent in other orders of organisms, such as anoxia-tolerance [6] and central nervous system regeneration [7]. Elucidating the mechanisms underlying these traits may provide key insights into therapeutic strategies for humans. Also, as a widely-distributed organism in freshwater ecosystems all over the world, $L$. stagnalis has recently been adopted as the model species of a new OECD test guideline [8]. It has been used as a biomonitor for the effects of a variety of water conditions and pollutants, including heavy metals $[9,10]$, water acidification [11], and pesticide use [12, 13]. Finally, L. stagnalis is relatively closely related to the land snail Biomphalaria glabrata, a vector for Schistosoma mansoni, the parasite that is the cause for schistosomiasis [14], a prominent public health burden in developing countries. Infection by $S$. mansoni elicits significant changes in neuropeptide dynamics in the B. glabrata CNS [15]. Interestingly, L. stagnalis is shown to be resistant to S. mansoni infection [16], suggesting that it may provide clues into strategies for preventing $S$. mansoni transmission by $B$. glabrata. In each case, thorough molecular characterization of the L. stagnalis CNS is key to realizing the translational potential of this model organism.

As ion channels are the fundamental units of excitability and synaptic transmission in the nervous system, characterization and identification of ion channels is enormously important to the field of neuroscience. Mutations in the genes encoding ion channels, or encoding proteins that regulate ion channels, have dramatic effects on normal functioning, resulting in a wide range of debilitating diseases [17, 18]. Therefore, thorough molecular characterization of ion channels has enormous basic and translational impacts on our understanding of the nervous system and beyond, necessitating characterization of ion channels in newly sequenced transcriptomes of model organisms such as L. stagnalis.

Animals have evolved a broad diversity of ion channels. In mammals, approximately 80 genes encode potassium channel subunits, forming 4 families and 7 subfamilies of potassium channels [19-21]. Though sodium and calcium channels are less numerous, their functional diversity is also rich. The diversity of the 5 classes of voltage-gated calcium channels is conferred by different $\alpha, \beta, \delta, \gamma$ subunit composition, while splice variation of those subunits gives rise to varied kinetics and pharmacology [22]. Furthermore, diversity of mammalian sodium channels is conferred by a variety of $\alpha$ - and $\beta$-subunits [23]. Finally, ligand-gated ion channels, though having considerably less variation, still vary in their permeabilities, kinetics, ligands, and subunit compositions [24]. They include ionotropic glutamate receptors [25], $\mathrm{GABA}_{\mathrm{A}}$ receptors [26], nicotinic acetylcholine receptors [27], ionotropic serotonin 5-HT3 receptors [28], and purinergic receptors [29].

Though the kinetics, pharmacology, permeability, and conductivity of the various ion channels are diverse, many ion channels are widely evolutionarily conserved. One of the earliest potassium channels to be characterized in Drosophila, the Shaker K+ channel, now known as Kv1.3, shares $82 \%$ sequence homology with the rat homologue [30]. This refrain of invertebrate channels being conserved in mammals is repeated in the mouse homologues of Drosophila Shaker, Shal, Shab, and Shaw $\mathrm{K}^{+}$channels, and in humans as well, with the Drosophila $\mathrm{K}+$ channels all having been shown to be expressed in human cardiac tissue [31]. On the molecular level, the segments of potassium channels that are evolutionarily conserved across vertebrates and invertebrates are those that are most important for channel function: the voltage gate, the selectivity filter, and the segment of the pore that gives the pore its shape [32]. This indicates that evolutionarily conserved ion channels are essential for fundamental processes in neurobiology.

The first ion channel to be cloned from $L$. stagnalis was the $\mathrm{GABA}_{\mathrm{A}}$ receptor, an ionotropic receptor and ligand-gated ion channel [33]. Since then, other evolutionarily conserved ion channels have been cloned from $L$. stagnalis, including glutamate receptor subunits [34-36], acetylcholine receptor [37], L-type, P/Q-type, N-type, R-type voltage-gated calcium channels and NALCN-like sodium leak channel [38-40], T-type voltage-gated calcium channels [41], and P2X receptor [42]. Fellow mollusc Aplysia californica, an immensely important model organism for neuroscience, has been used extensively to study ion channels [43]. Study of its neuronal transcriptome has revealed expression of a variety of ion channels, including ionotropic glutamate, acetylcholine, and GABA/glycine receptors, voltagegated $\mathrm{Ca}^{2+}$ and $\mathrm{Na}^{+}$channels, several families of $\mathrm{K}^{+}$ channels, amiloride-sensitive $\mathrm{Na}^{+}$channels, cyclic nucleotide-gated channels, and inositol trisphosphate/ ryanodine receptors [44]. Another molluscan model organism, Biomphalaria glabrata, has been shown to express transcripts encoding various ion channels as well, including $\mathrm{Na}^{+}, \mathrm{K}^{+}, \mathrm{Cl}^{-}, \mathrm{Ca}^{2+}$, and TRP cation channels, as well as glutamate, acetylcholine, and GABA receptors 
[45]. Furthermore, novel, evolutionarily conserved ion channels have been first identified and characterized in various invertebrate species. For instance, the first hyperpolarization-activated cyclic nucleotide-gated (HCN) channel was cloned from sea urchin sperm [46], leading to the discovery of several mammalian homologues. Furthermore, D. melanogaster has been used to identify the molecular determinants of the newest family of ion channels, calcium release-activated current (CRAC) channels $[47,48]$. This demonstrates that invertebrate models, including $L$. stagnalis, continue to be a rich resource of discovery for evolutionarily conserved fundamental neuronal signaling processes applicable to all organisms.

Recent advances in high-throughput RNA sequencing (RNAseq) have much accelerated the search for novel ion channel and receptor families. Current sequence information for the L. stagnalis CNS is obtained from EST libraries [49] and a de novo RNA-seq assemblies [50]. However, due to the low coverage of EST libraries and fragmented nature of de novo assemblies, much remains to be characterized about the L. stagnalis CNS transcriptome. The recent availability of a preliminary L. stagnalis reference genome [51] has provided the opportunity to

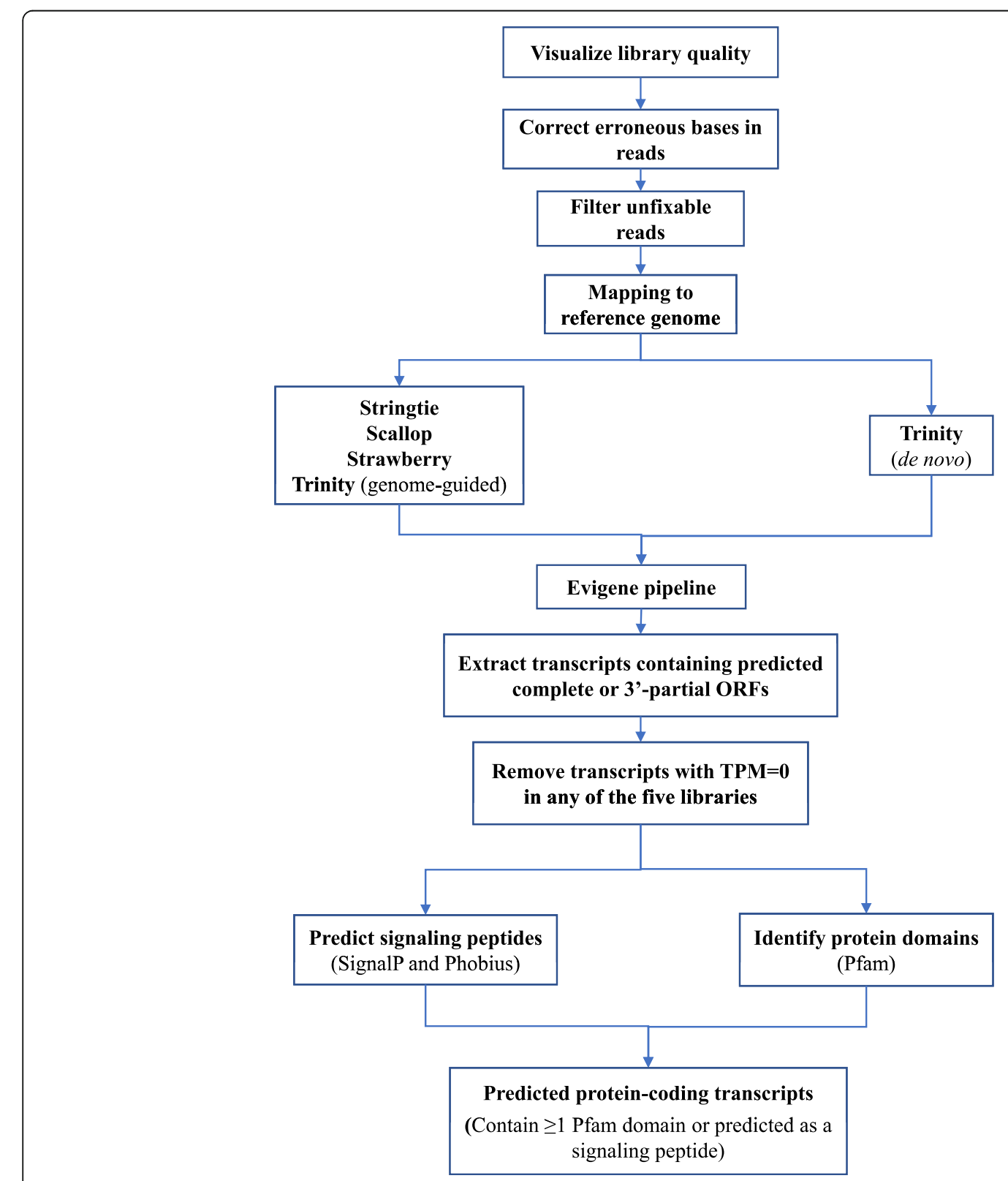

Fig. 1 Workflow of quality control, assembly and prediction of protein-coding transcripts in the L. stagnalis CNS 
Table 1 BLAST hit of the top 20 expressed transcripts in the L. stagnalis CNS

\begin{tabular}{|c|c|c|c|c|}
\hline Accession & Gene name & Species & Identity (\%) & Description \\
\hline BAW32915.1 & $\begin{array}{l}\text { Cytochrome c oxidase subunit l, partial } \\
\text { (mitochondrion) }\end{array}$ & Ringicula cf. pilula & 84 & Energy production \\
\hline AAB29129.1 & preproLYCP & Lymnaea stagnalis & 99.1 & \\
\hline P42579.1 & Sodium-influx-stimulating peptide & Lymnaea stagnalis & 100 & \\
\hline \multicolumn{4}{|c|}{ Predicted signaling peptide } & Neuropeptide signaling \\
\hline P80090.2 & Molluscan insulin-related peptide 3 & Lymnaea stagnalis & 99.2 & \\
\hline ABV22501.1 & Myoglobin & Biomphalaria tenagophila & 69.5 & Oxygen transport \\
\hline \multicolumn{4}{|c|}{ Predicted signaling peptide } & Neuropeptide signaling \\
\hline P58154.1 & Acetylcholine-binding protein & Lymnaea stagnalis & 98.3 & Synaptic transmission \\
\hline \multicolumn{4}{|c|}{ Predicted signaling peptide } & Neuropeptide signaling \\
\hline P42577.2 & Soma ferritin & Lymnaea stagnalis & 100 & Metal ion homeostasis \\
\hline YP_006665701.1 & Cytochrome c oxidase subunit III (mitochondrion) & Galba pervia & 85 & Energy production \\
\hline AAD02473.1 & Cardioexcitatory peptide precursor & Lymnaea stagnalis & 94.1 & Neuropeptide signaling \\
\hline AAS20460.1 & Granularin & Lymnaea stagnalis & 100 & Defense response \\
\hline \multicolumn{4}{|c|}{ Predicted signaling peptide } & Neuropeptide signaling \\
\hline P48416.1 & Cytochrome P450 & Lymnaea stagnalis & 96.3 & Oxidoreductase activity \\
\hline XP_012943264.1 & $\begin{array}{l}\text { PREDICTED: uncharacterized protein } \\
\text { LOC106013068 }\end{array}$ & Aplysia californica & 45.2 & \\
\hline P06308.2 & Ovulation prohormone & Lymnaea stagnalis & 87.4 & \\
\hline \multicolumn{4}{|c|}{ Predicted signaling peptide } & Neuropeptide signaling \\
\hline ARS01367.1 & Ffamide 2 & Deroceras reticulatum & 58.2 & \\
\hline \multicolumn{5}{|c|}{ Predicted signaling peptide } \\
\hline
\end{tabular}

enhance the completeness and coverage of the CNS transcriptome through genome-guided assembly of RNA-seq reads. This can potentially allow identification of novel L. stagnalis transcripts and characterization of whole protein classes, particularly of ion channels and ionotropic receptors, which have not yet been fully characterized. In this study, we employed a combination of genome-guided and de novo assembly programs to create an assembly that improves upon the completeness and coverage of existing resources. We have also identified transcripts encoding ion channels and ionotropic receptors and compared them to other species. This provides the most thorough characterization to date of the CNS transcriptome of this important molluscan model organism and builds a crucial foundation for leveraging the unique advantages of $L$. stagnalis in a wide range of research fields. Furthermore, as this study characterizes ion channels in an invertebrate molluscan species, where essential neurobiological processes are likely to be evolutionarily conserved across species, this study serves as a potential starting point for the identification of novel ion channel families which may be evolutionarily conserved in mammals.

\section{Results}

\section{CNS transcriptome assembly and annotation}

L. stagnalis is a widely used model organism in understanding the fundamental mechanisms of neural function due to its simple and well-characterized CNS. A de novo assembly of the L. stagnalis CNS transcriptome from $100 \mathrm{bp}$ single-end reads has previously been published [50], but the completeness of the assembly had been hindered by the lack of a genome reference. With the aid of a preliminary, recently sequenced and

Table 2 BUSCO analyses of protein-coding sequences identified in the published EST library and de novo assembly, the current assembly and combined set

\begin{tabular}{lllll}
\hline & Complete (single-copy) & Complete (duplicated) & Fragmented & Missing \\
\hline Current assembly & $\mathbf{8 0 3}(82.1 \%)$ & $\mathbf{1 0 4}(10.6 \%)$ & $\mathbf{3}(0.3 \%)$ & $\mathbf{6 8}(7.0 \%)$ \\
De novo assembly & $\mathbf{8 8 5}(90.5 \%)$ & $\mathbf{1 0}(1.0 \%)$ & $\mathbf{1 3}(1.3 \%)$ & $\mathbf{7 0}(7.2 \%)$ \\
EST library & $\mathbf{1 2 4}(12.7 \%)$ & $\mathbf{7}(0.72 \%)$ & $\mathbf{6 1}(6.2 \%)$ & $\mathbf{7 8 6}(80.4 \%)$ \\
Combined & $\mathbf{9 0 7}(92.7 \%)$ & $\mathbf{3 4}(3.5 \%)$ & $\mathbf{3}(0.3 \%)$ & $\mathbf{3 4}(3.5 \%)$ \\
\hline
\end{tabular}


assembled $L$. stagnalis genome, here we employed a combination of genome-guided and de novo approaches to assemble reads from four $150 \mathrm{bp}$ paired-end libraries that we prepared from four adult central ring ganglia and the aforementioned published $100 \mathrm{bp}$ single-end library (Sadamoto et al. 2012) to create a new and improved L. stagnalis CNS transcriptome assembly (Fig. 1). After correcting for erroneous bases and removing unfixable reads (Table S3), each of the five libraries had at least $88 \%$ of the reads mapped to the genome with unique location (Table S4). The mapped reads in each library were assembled in a genome-guided manner using multiple assemblers and the unmapped reads were pooled for de novo assembly using Trinity. Altogether, this resulted in 22 assemblies containing 1,651,924 transcripts in total (Table S5). To identify putative protein-coding transcripts (Fig. 1), we analyzed this set of sequences using the Evigene pipeline to identify 196, 514 non-redundant transcripts that contained a complete or 3'-partial (containing start but not stop codon) open reading frame (ORF) (Table S6). Potential transcript artifacts and noise were filtered out, resulting in a transcript set containing 68,094 transcripts. As ORFs can arise spuriously [52], we further identified protein domain-containing transcripts by annotation against the Pfam database and/or signaling peptide prediction by both SignalP and Phobius, resulting in 17,832 sequences as the final set of predicted protein-coding

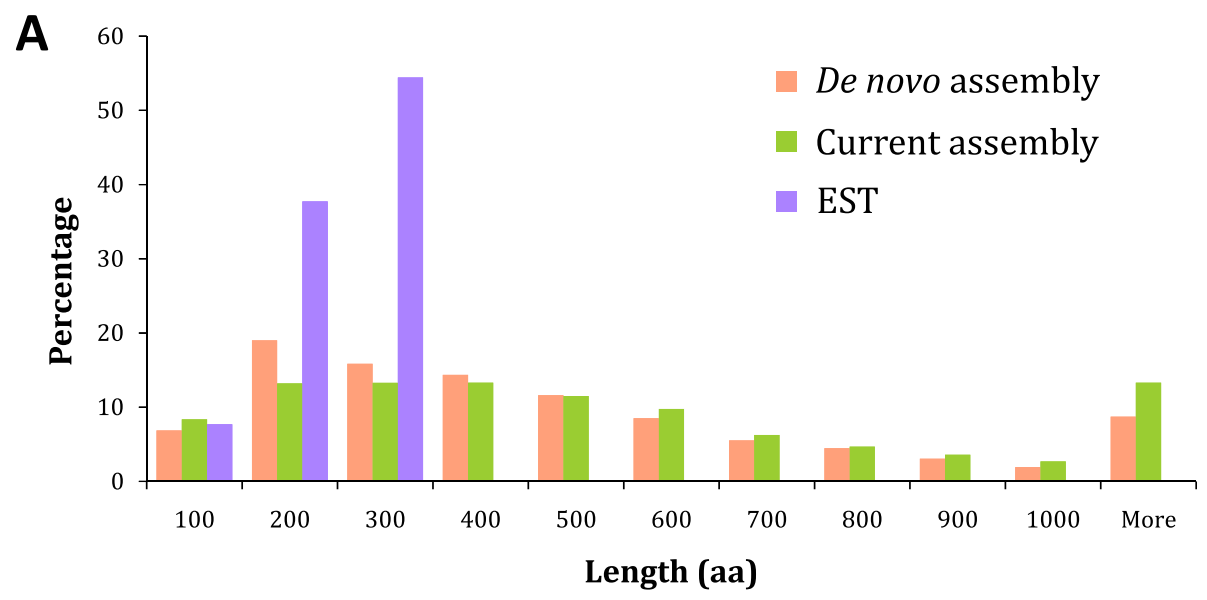

B

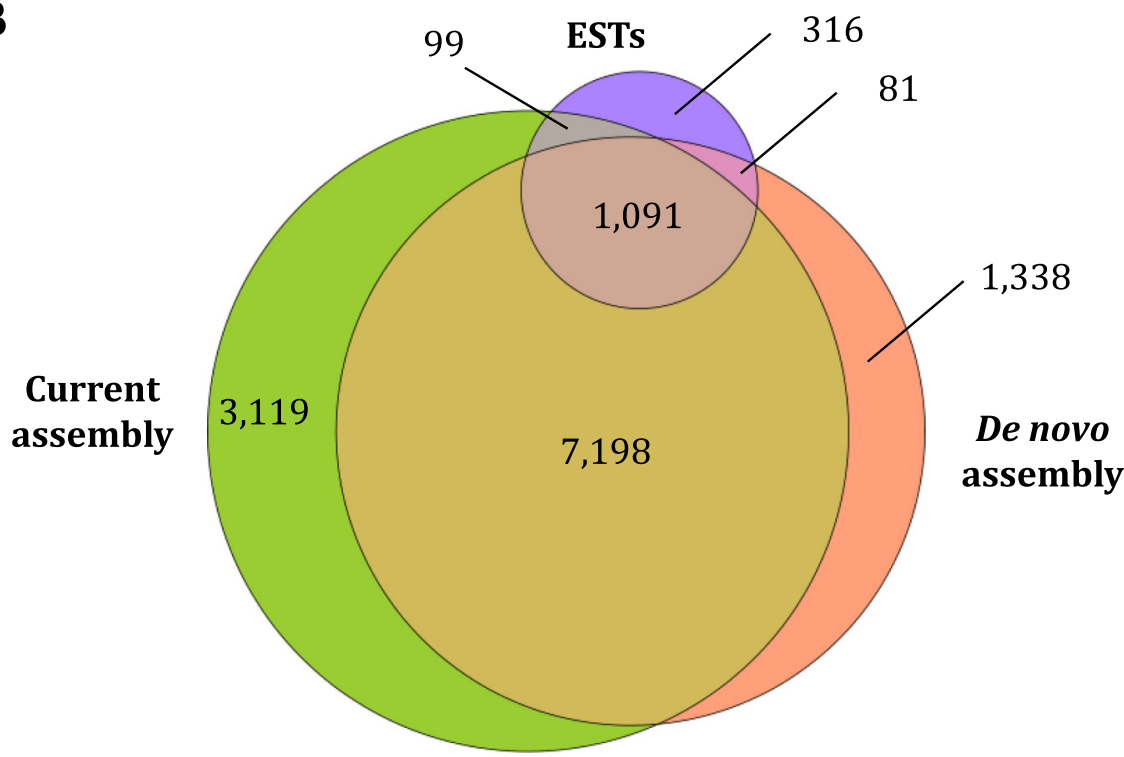

Fig. 2 Comparison of predicted L. stagnalis protein-coding transcripts identified in the current assembly with those identified in the previously published EST library [49] and de novo assembly [50]. a Distribution of translated amino acid sequence lengths of predicted protein-coding transcripts as a percentage of the total number of transcripts in previous and current assemblies. The current assembly contains a greater percentage of longer transcripts than previous assemblies. b Overlapping and distinct Nr database hits found in predicted protein-coding sequences in previous and current assemblies. The current assembly defines a greater number of new and distinct hits than previous assemblies 
transcripts. Annotation of the top 20 most highly expressed predicted protein-coding transcripts showed that the majority were annotated or predicted as signaling peptides (Table 1).

\section{Comparison and aggregation of current assembly with previously published $L$. stagnalis CNS transcriptome}

To compare the number and quality of predicted protein-coding transcripts uncovered in our current assembly with those in the previously published expressed sequence tags (EST) library [49] and de novo assembly [50], these two earlier sets of sequences were processed as described above to identify potential protein coding transcripts. We found 1946 sequences (out of 10,375 in total) that contained at least one protein domain and/or were annotated as a signaling peptide by both Phobius and SignalP in the EST library, and 11,742 such sequences (out of 116,355 in total) in the de novo assembled library. BUSCO analysis showed that the predicted protein-coding transcripts identified in the current assembly contained $92.7 \%$ of single-copy ortholog genes present in $>90 \%$ eukaryotic species, higher than the de novo assembly (91.5\%) and the EST library (13.4\%) (Table 2).

Comparison of the amino acid sequence length across the three sets of predicted protein coding sequences (Fig. 2a) found that the mean (ESTs: 196 aa; de novo:
470 aa; current: 569 aa), median (ESTs: 209 aa; de novo: 353 aa; current: 415 aa), and maximum (ESTs: 313; de novo: 8195 aa; current: 13,109 aa) sequence lengths in the current assembly were all higher than those in the ESTs library and de novo assembly. Comparison of unique $\mathrm{Nr}$ hits in the three sets of predicted proteincoding sequences showed that $7198 \mathrm{Nr}$ hits were found in all three, with 3119,1338 , and 316 hits found only in the current assembly, de novo assembly, and ESTs library, respectively (Fig. 2b). Taken together, these findings indicate that the current combined genome-guided and de novo assembly improved the coverage and sequence length of the $L$. stagnalis CNS protein-coding transcriptome.

As shown in Fig. 2b, each of the three transcriptome libraries captured sequences that were absent from the other two. Therefore, we combined the three sets of sequences $(31,520$ sequences in total) to create the most comprehensive predicted protein-coding sequences library to date. These sequences were further clustered into a collection of 16,447 non-redundant predicted protein-coding sequences. BUSCO analysis showed that this set contains $96.2 \%$ of 978 single-copy orthologs present in $>90 \%$ of all eukaryotic species, higher than all three libraries when analyzed individually (Table 2). We next examined this comprehensive set of sequences to characterize gene expression in the L. stagnalis CNS.

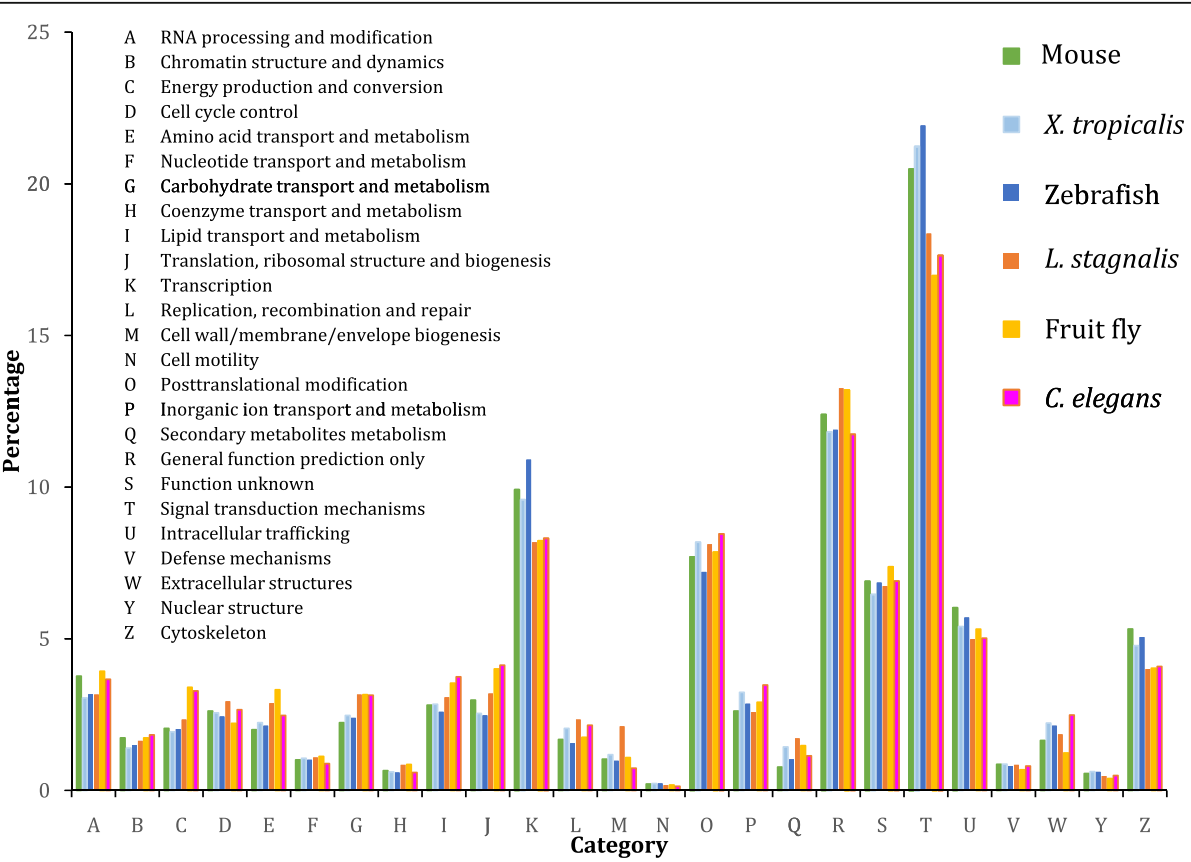

Fig. 3 Comparison of KOG annotations of protein-coding transcripts expressed in the CNS of key vertebrate and invertebrate neuroscience model organisms (E-value <1E-5). Markedly, compared to invertebrates, vertebrate model organisms display increased percentage of transcripts involved in transcription, intracellular trafficking, and cytoskeleton. Meanwhile, compared to vertebrates, invertebrate model organisms display increased percentage of transcripts involved in energy production and conversion, amino acid transport and metabolism, carbohydrate transport and metabolism, and lipid transport and metabolism 


\section{Comparisons of CNS transcriptomes of key neuroscience model organisms}

This improved L. stagnalis CNS transcriptome provides an unprecedented opportunity to carry out comparative studies of CNS gene expression in a number of commonly used vertebrate and invertebrate neuroscience model organisms, so as to gain insights into commonalities and differences that may inform future studies. To this end, we analyzed publicly deposited RNA-seq libraries to characterize gene expression in the CNS of adult Mus musculus (mouse) [53], Xenopus tropicalis [53], Danio rerio (zebrafish) [54], Drosophila melanogaster (fruit fly) (BioProject: PRJNA320764), and Caenorhabditis elegans [55] (Table S1). Due to differing levels of noncoding RNA annotation for each species, we have focused our analyses on protein-coding transcripts. Annotation of the top 20 expressed transcripts in each of these five species (Tables S7, S8, S9, S10 and S11), in the same manner as done for $L$. stagnalis (Table 1), found a predominance of transcripts involved in energy production, protein synthesis, and signaling, in both vertebrate and invertebrate species. To gain a complementary perspective, we characterized the functional categorization of CNS protein-coding transcripts amongst $L$. stagnalis and these five common model organisms (Fig. 3) using their KOG annotations. Interestingly, the general distribution was largely similar, with "Signal transduction mechanisms" being the most abundant category overall. Nevertheless, in all three of the vertebrate species examined, more transcripts were functionally classified as "Signal transduction mechanisms", "Transcription", and "Cytoskeleton" than in the three invertebrate species. Conversely, in the three invertebrate species, more transcripts were identified to be functionally involved in "Carbohydrate transport and metabolism", "Lipid transport and metabolism", and "Translation, ribosomal structure and biosynthesis" than those in the three vertebrate species.

We further clustered CNS protein-coding transcripts in the six species into orthologous groups to identify

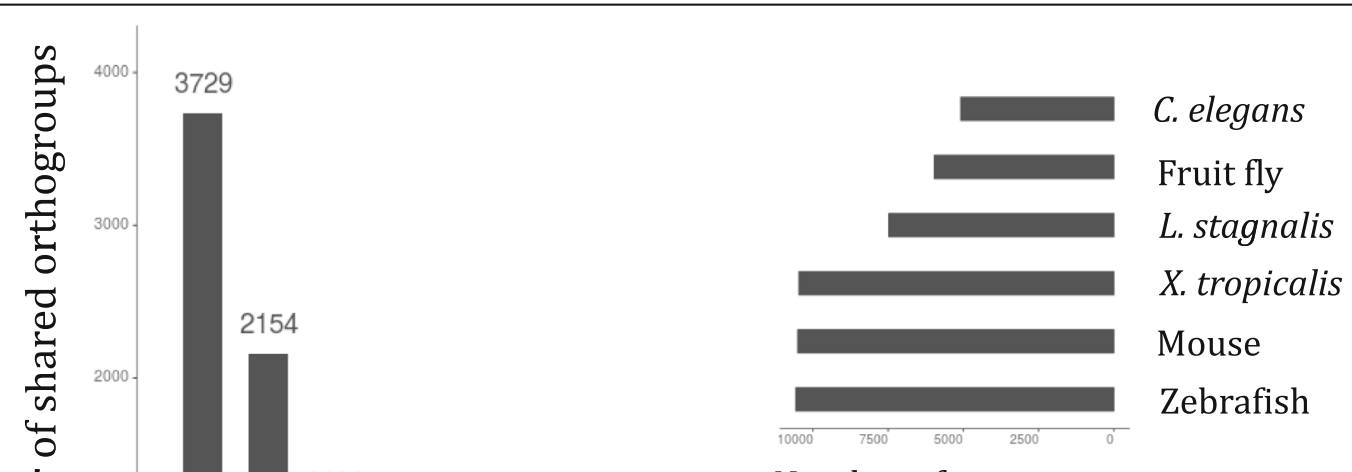

Number of orthogroups

C. elegans Fruit fly

\section{L. stagnalis}

X. tropicalis

\section{Mouse}

Zebrafish
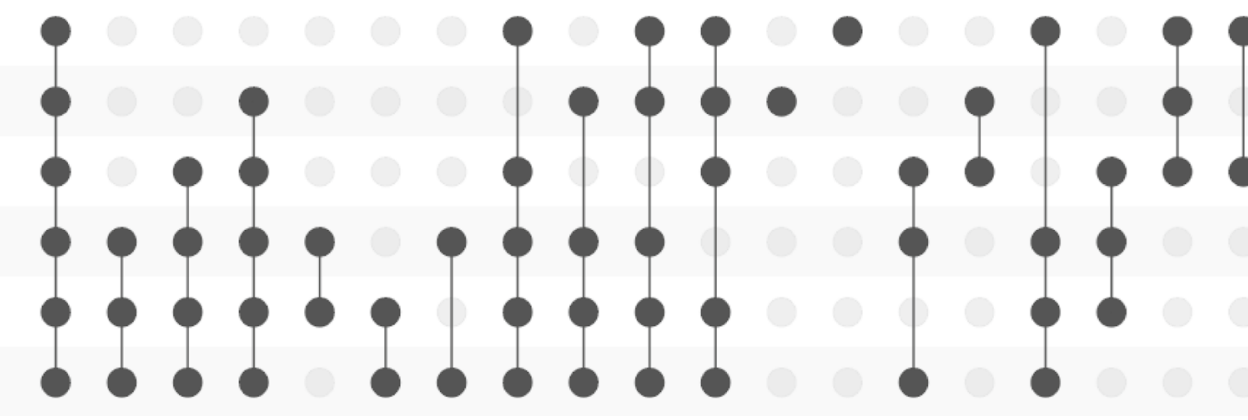

Fig. 4 Membership of species in the orthogroups identified amongst protein-coding transcripts expressed in the CNS of M. musculus, X. tropicalis, D. rerio, L. stagnalis, D. melanogaster, and C. elegans. For clarity, only the 20 largest sets of shared orthogroups are shown. Dark and grey circles represent presence and absence, respectively, of the given species in each set of shared orthogroups. The number of orthogroups that each species is present in is shown in the inset bar graph. All species share the greatest number of orthogroups, followed by all vertebrates. Strikingly, of all three invertebrate species, L. stagnalis shares the greatest number of orthogroups with the three vertebrate species (1111) 


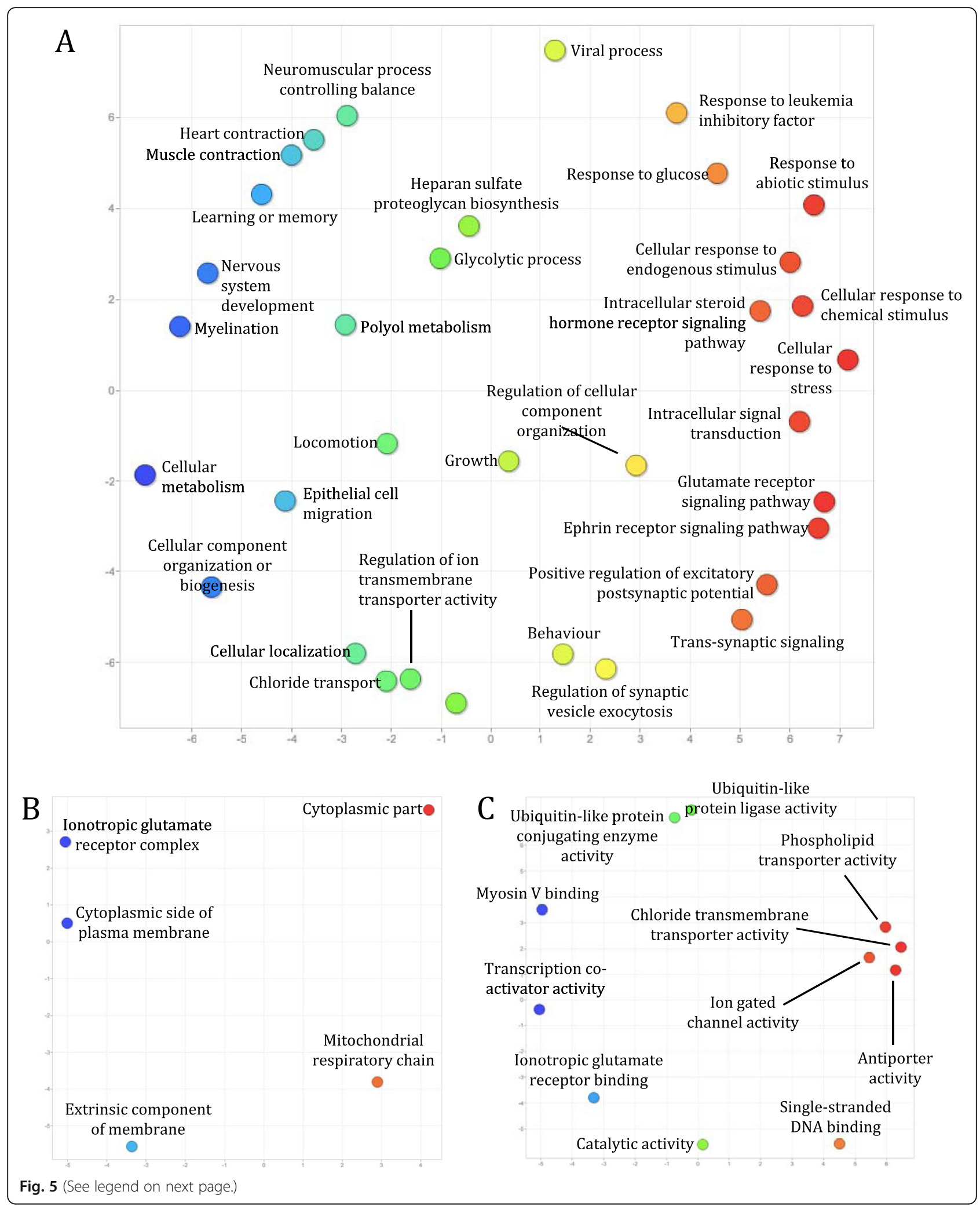


(See figure on previous page.)

Fig. 5 Enriched GO terms of genes encoded by transcripts in the 3729 orthogroups shared amongst M. musculus, X. tropicalis, D. rerio, L. stagnalis, D. melanogaster, and C. elegans. Using the REVIGO tool, GO terms in the "Biological process" (a), "Cellular components" (b) and "Molecular function" (c) classes were clustered in two-dimensional space via the SimRel semantic similarity measure to summarize their relationship to each other and coloured according to their positions on the semantic x-axis. Consequently, related GO terms are closer on the plot and more similar in colour. The arbitrary semantic space units on either axis have no intrinsic meaning. Orthogroups common amongst all six organisms commonly involved a intracellular signaling pathways (red/orange), b ionotropic glutamate receptor signaling (blue), and c ion transport (red/orange), among others

core CNS functions that are mediated by orthologous genes across distantly related species. In total, we identified 12,022 orthogroups, with 3729 orthogroups containing genes from all of the six species examined. Interestingly, L. stagnalis shares more orthogroups with the three vertebrate species than with $C$. elegans or fruit fly (Fig. 4). Functional enrichment of mouse genes (as they have the best annotation quality) in orthogroups shared amongst all species identified a variety of key CNS functions, including "Myelination" (GO:0042552), "Glutamate receptor signaling pathway" (GO:0007215), "Mitochondrial respiratory chain" (GO:0005746), and "ionotropic glutamate receptor binding" (GO:0035255) (Fig. 5; Table S12). These findings were complemented by Reactome (Table S13) and KEGG (Table S14) pathway analyses of the same set of mouse genes, which further identified known core CNS functions including "Axon guidance" (REAC:R-MMU-422475), "Cellular response to hypoxia" (REAC: R-MMU-2262749), "cAMP signaling pathway" (KEGG:04024) and "Insulin signaling pathway" (KEGG:04910). Furthermore, we identified 222 transcription factors whose binding motifs were enriched in the promoter region of this set of mouse genes (Table S15), identifying both well-characterized neuronal transcription factors such as CREB1 and AP2, but also novel proteins that may provide targets of future studies. We also performed similar enrichment analyses of genes in the 2154 vertebrate-only and 88 invertebrate-only orthogroups, in order to gain insights into differential functional specializations of the vertebrate and invertebrate CNS. Enrichment of mouse genes in the vertebrate-only orthogroups showed a constellation of GO (Fig. 6; Table S16), Reactome (Table S17) and KEGG (Table S18) terms related to immune/inflammatory processes, including "Leukocyte chemotaxis" (GO: 0030595), "Regulation of interferon-beta production" (GO:0032648), "Complement and coagulation cascades" (KEGG:04610), and "Receptor-type tyrosine-protein phosphatases" (REAC:R-MMU-388844). Enrichment of fruit fly genes in the invertebrate-only orthogroups found terms relating to response to chemical stimuli, such as "Sensory perception of chemical stimulus" (GO: 0007606), "Peptide receptor activity" (GO:0001653), and "G-protein coupled peptide receptor activity" (GO: 0008528) (Fig. 7; Table S19). No enriched Reactome or KEGG pathways were found for this group of genes.

\section{Identification of ion channels and ionotropic receptors expressed in the $L$. stagnalis CNS and interspecies comparison}

Ion channels and ionotropic neurotransmitter receptors play critical roles in regulating neuronal excitability and synaptic transmission in the CNS. As the simple and well-characterized L. stagnalis CNS is widely employed to study the fundamental mechanisms of neuronal function and circuit function [4,56-58], we next sought to identify ion channels and ionotropic receptors expressed in the L. stagnalis CNS. We identified 211 proteincoding transcripts that contained both 1) ion channel or ionotropic receptor protein domains and 2) transmembrane helices to characterize the complement of ion channels and ionotropic receptors expressed in the $L$. stagnalis CNS. A proportion of sequences in this set were substantially shorter or longer than their best matched sequence in the $\mathrm{Nr}$ database. However, for the sake of completeness, we have included all in our analysis as some may be informative as splice isoforms. Sequence alignment-based phylogenetic analysis of these transcripts showed that they encode a wide range of ionotropic neurotransmitter receptors (Fig. 8; Tables S20, S21 and S22), $\mathrm{K}^{+}$(Fig. 9; Table S23), $\mathrm{Ca}^{2+}$ (Fig. 10; Table S24), $\mathrm{Na}^{+}$(Fig. 11; Table S25), $\mathrm{Cl}^{-}$(Fig. 12; Table S26), cation (Fig. 13; Table S27), and transient receptor potential (TRP) (Fig. 14; Table S28) channels. Only 31 of the 211 putative ion channel/ionotropic receptor transcripts were annotated as a L. stagnalis sequence in the $\mathrm{Nr}$ database, indicating that majority of the sequences were identified here in this species for the first time.

We further examined the degrees of protein sequence similarity between ion channel/ionotropic receptor transcripts identified in L. stagnalis and those in these five model organisms through the BLASTp program and resulting bitscores of BLASTp hits. We plotted the standardized bitscore of each $L$. stagnalis ion channel/receptor against its top BLASTp hit in each species in a Clustergrammer heatmap (link provided in the figure legend), where bitscores lower and higher than the average are shown in blue and red, respectively (Fig. 15). Each row corresponds to a putative L. stagnalis ion channel/ ionotropic receptor transcript and each column is one of the five species examined. First, ranking the species (columns) by the absolute sum of standardized bitscores, such that the species with the highest degree of 


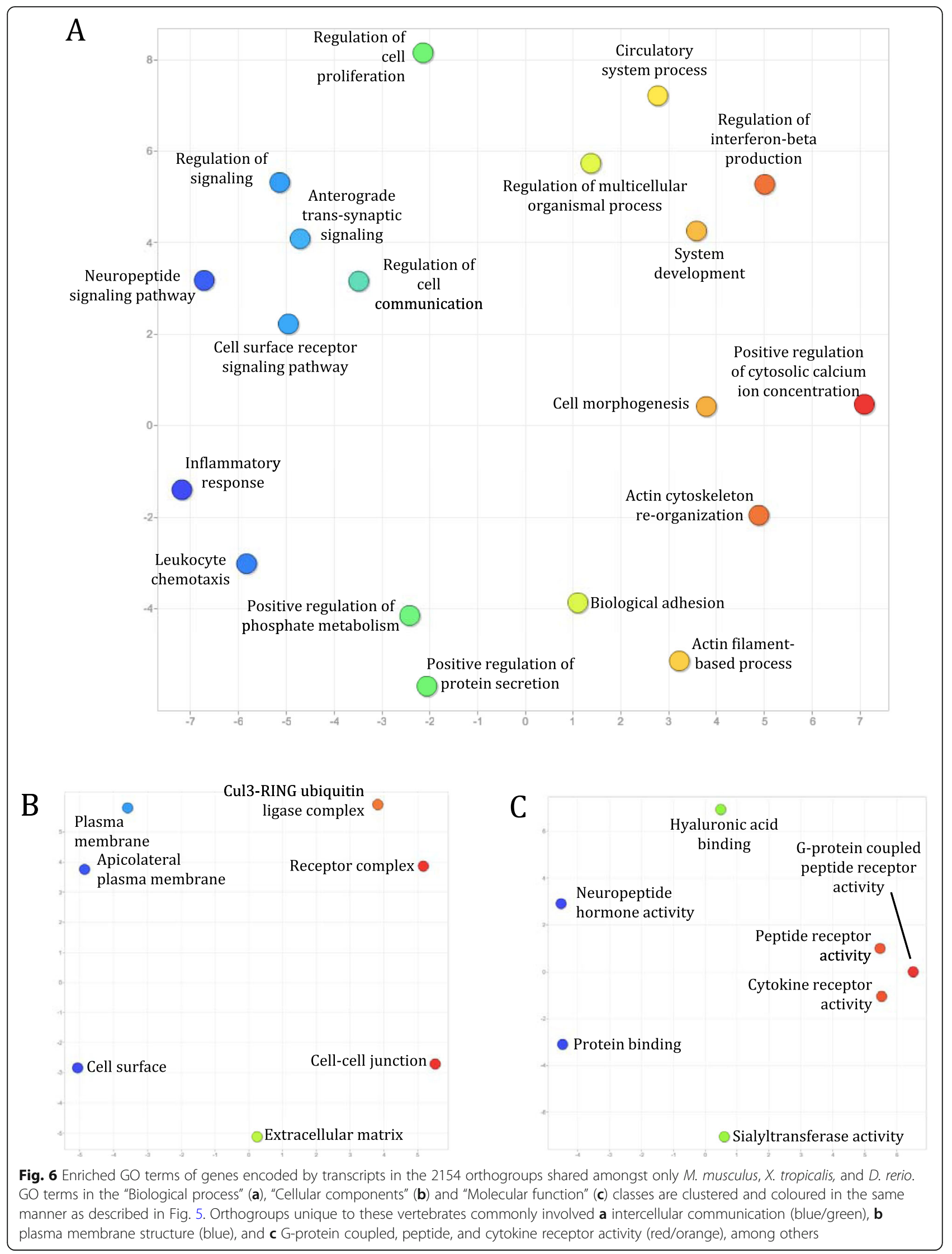




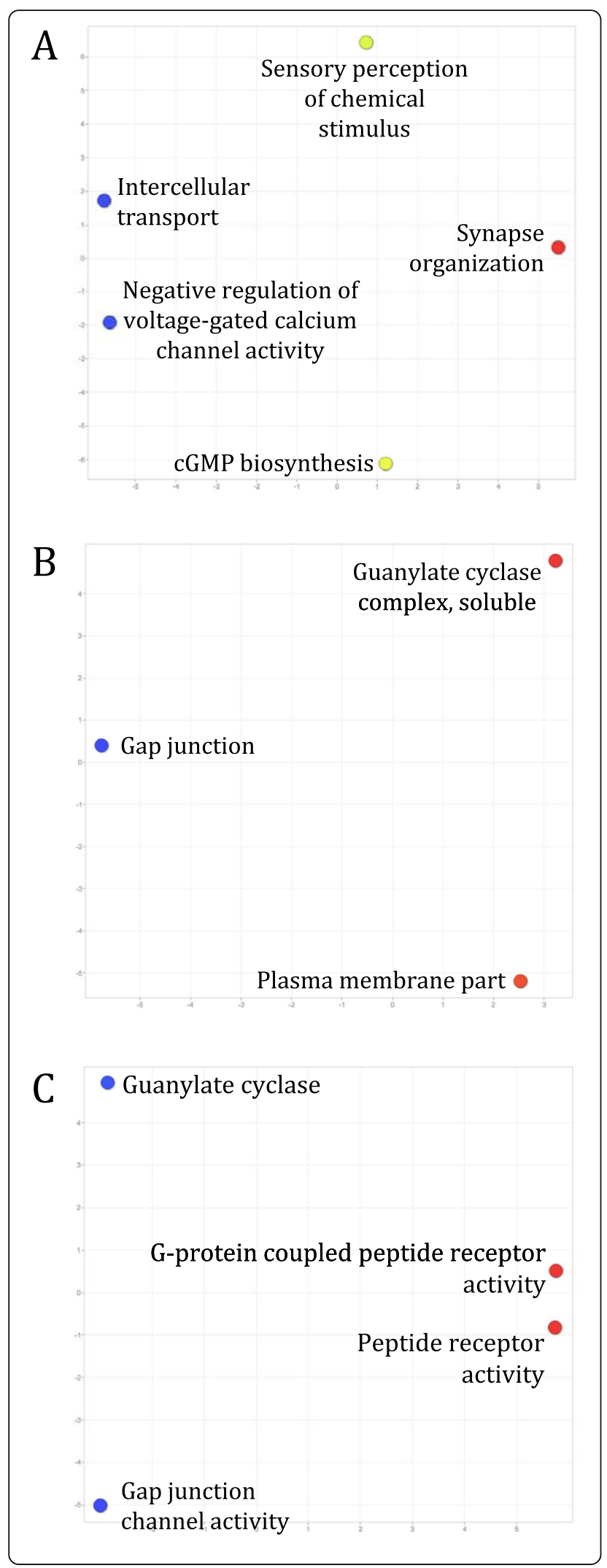

Fig. 7 Enriched GO terms of genes encoded by transcripts in the 88 orthogroups shared amongst only L. stagnalis, D. melanogaster, and C. elegans. GO terms in the "Biological process" (a), "Cellular components" (b) and "Molecular function" (c) classes are clustered and coloured in the same manner as described in Fig. 5.

Orthogroups unique to these invertebrates commonly involved $\mathbf{a}$ voltage-gated calcium channel activity regulation (blue), b plasma membrane structure (red), and c G-protein coupled peptide and peptide receptor activity, among others

sequence similarity with $L$. stagnalis is on the left of the graph, we observed that putative L. stagnalis ion channel/ionotropic receptor transcripts showed the most sequence similarity with their homologs in fruit fly and $X$. tropicalis, and the least with mouse and $C$. elegans (Fig. 15A). Then, by sorting the transcripts according to their class (ex. $\mathrm{Ca}^{2+}$ channels, $\mathrm{K}^{+}$channels, glutamate receptors, etc.), we found that $L$. stagnalis transcripts encoding $\mathrm{Ca}^{2+}$ channels showed the highest degrees of sequence similarities with their homologs as compared to all other classes (Fig. 15A). Finally, to more closely examine the individual $L$. stagnalis transcripts that showed the highest sequence similarities with other species, we sorted the transcripts (rows) by their absolute bitscore sum and focused on the top 20 transcripts (Fig. 15B). Indeed, in this list we observed numerous putative $\mathrm{Ca}^{2+}$ channel transcripts, such as ryanodine receptors (RyR), inositol 1,4,5-trisphosphate receptors, and voltage-gated $\mathrm{Ca}^{2+}$ channels (both $\mathrm{L}$ - and non-L-type). However, there were also a diversity of cation, $\mathrm{K}^{+}$and TRP channels in this list. Taken together, these findings provide not only a resource for investigating the molecular mechanisms of CNS neural transmission and neuronal excitability in L. stagnalis, but also a foundation for better understanding the conservation and evolution of these processes across species.

\section{Discussion}

L. stagnalis possesses a simple and well-characterized CNS that has been widely employed to elucidate key mechanisms of neural function [2, 4, 59-63], response to environmental pollutants $[9,10,13]$, and resistance to parasitic infection [64]. However, molecular characterization of the CNS has been impeded by the lack of a comprehensive transcriptome. Here, we carried out the first genomeguided assembly of the $L$. stagnalis CNS transcriptome and integrated it with existing sequence databases to create the most comprehensive to-date transcriptome resource.

We took a combination of approaches to improve upon the existing sequence information. Firstly, we performed strand-specific paired-end RNA-seq that produced $150 \mathrm{bp}$ long reads in this study, as compared to the unstranded $100 \mathrm{bp}$ single-end reads used in the previously published de novo assembly [50]. This allows for 


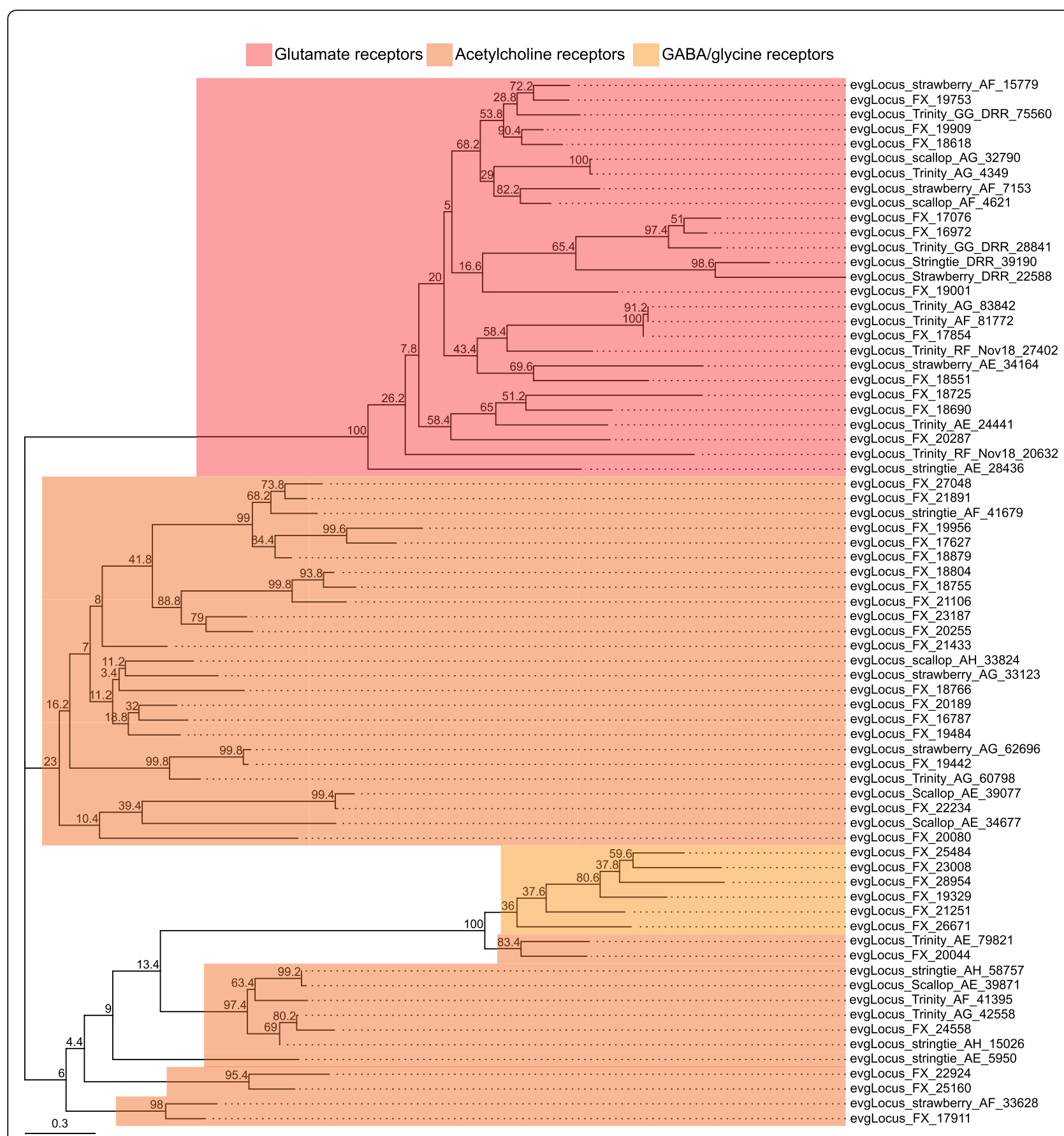

Fig. 8 lonotropic neurotransmitter receptor families identified in the L. stagnalis CNS transcriptome assembly. The tree with the highest log likelihood $(-7343.03)$ is shown. The percentage of trees in which the associated taxa clustered together (bootstrap values) is shown next to the branches. The tree is rooted at the mid-point and is drawn to scale, with branch lengths measured in the number of substitutions per site (see scale in bottom left). This analysis involved 33 amino acid sequences. There were a total of 133 positions in the final dataset. Evolutionary analyses were conducted in MEGA X (ver. 10.1.8) and tree visualization and annotation was conducted using the ggtree package (ver. 2.0.4) for $R$ (ver. 3.6.3). This L. stagnalis transcriptome assembly identifies a wide diversity of putative acetylcholine, GABA/glycine, and glutamate receptors. The accession numbers and BLAST information of transcripts represented in this tree are provided in Tables S20, S21 and S22

better unique mapping to the reference genome and splice junction detection, and thus more accurate reconstruction of transcripts [65]. Secondly, we leveraged the strengths of multiple assembly software packages and both genome-guided and de novo assembly methods, which have been shown to increase transcriptome completeness and limit sequence redundancy [66, 67]. We further added to the diversity of the final transcript set 


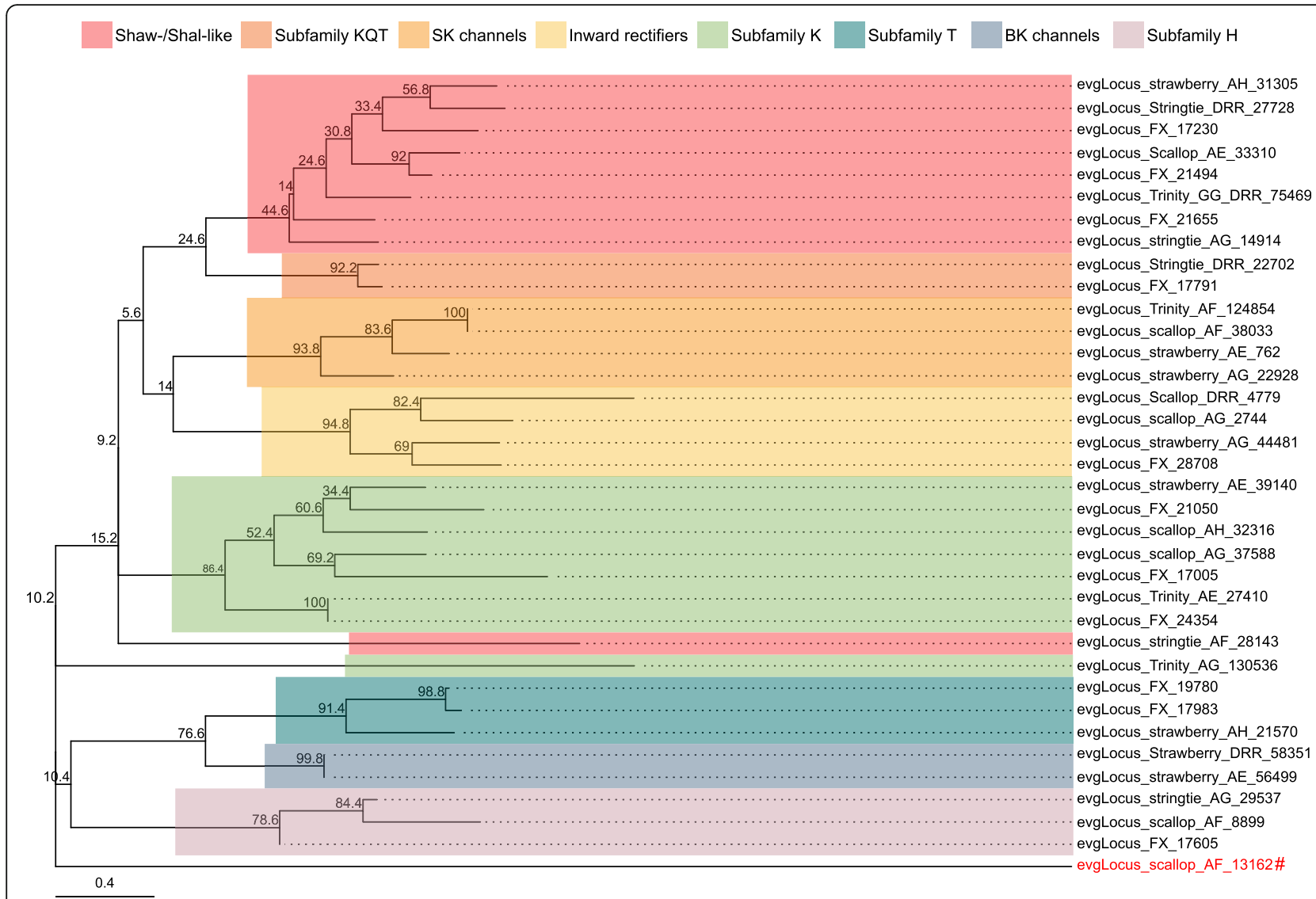

Fig. $9 \mathrm{~K}^{+}$channel subtypes and families identified in the L. stagnalis CNS transcriptome assembly. The tree was generated as described in Fig. 8, where the analysis involved 36 amino acid sequences and yielded a total of 75 positions in the final dataset, resulting in a tree with highest log likelihood of -4511.88 . The accession numbers and BLAST information of transcripts represented in this tree are provided in Table S23. A wide diversity of putative $L$. stagnalis $\mathrm{K}^{+}$channel subtypes and families are identified, including a potentially novel family of voltage-gated $\mathrm{K}^{+}$channels (denoted by red label, \#)

by including previously published ESTs [49] and de novo assembled transcripts [50]. Finally, we used a combination of ORF detection, expression-based filtering, protein domain identification and clustering methods to remove sequencing/assembly artefacts and fragmented/ redundant sequences in this aggregated set, creating a collection of high-confidence predicted protein-coding transcripts (available on http://lymnaea.org). Indeed, we have shown that this transcript set exceeds existing resources in terms of sequence length, completeness, and diversity (Fig. 2, Table 2).

In this study, we functionally characterized the L. stagnalis CNS transcriptome and identified transcripts critical for electrical excitability and neurotransmission. First, we demonstrated that annotated or predicted neuropeptides predominate the list of top 20 most abundantly expressed transcripts, which is consistent with the importance of neuropeptide signaling in regulating a variety of physiological processes in L. stagnalis, including neuronal excitability [68], heartbeat [69], feeding [70], and reproduction [71, 72]. This group of transcripts is strikingly similar to most highly expressed transcripts observed in the CNS of two other mollusc species, Tritonia diomedea [73] and Biomphalaria alexandrina [74], which also include transcripts encoding soma ferritin, acetylcholine-binding protein, and numerous neuropeptides such as sodium-influx-stimulating peptide, molluscan insulin-related peptide 3 and cardioexcitatory peptide. Then, we characterized the functional distribution of protein-coding transcripts expressed in the $L$. stagnalis CNS through KOG annotations (Fig. 3), showing that they are predominantly involved in mediating signaling transduction, general function and transcription processes. Finally, for the first time, we systematically identified transcripts that putatively encode 43 subtypes of $\mathrm{Na}^{+}, \mathrm{K}^{+}, \mathrm{Ca}^{2+}, \mathrm{Cl}^{-}$and cation ion channels and ionotropic neurotransmitter receptors (Figs. 8, 9, 10, 11, 12, 13 and 14, Tables S15, S16, S17, S18, S19, S20, S21, S22 and S23), greatly expanding our understanding of the molecular determinants of neural excitability and transmission in the L. stagnalis CNS. As the L. stagnalis CNS has been widely employed to study ion channels 


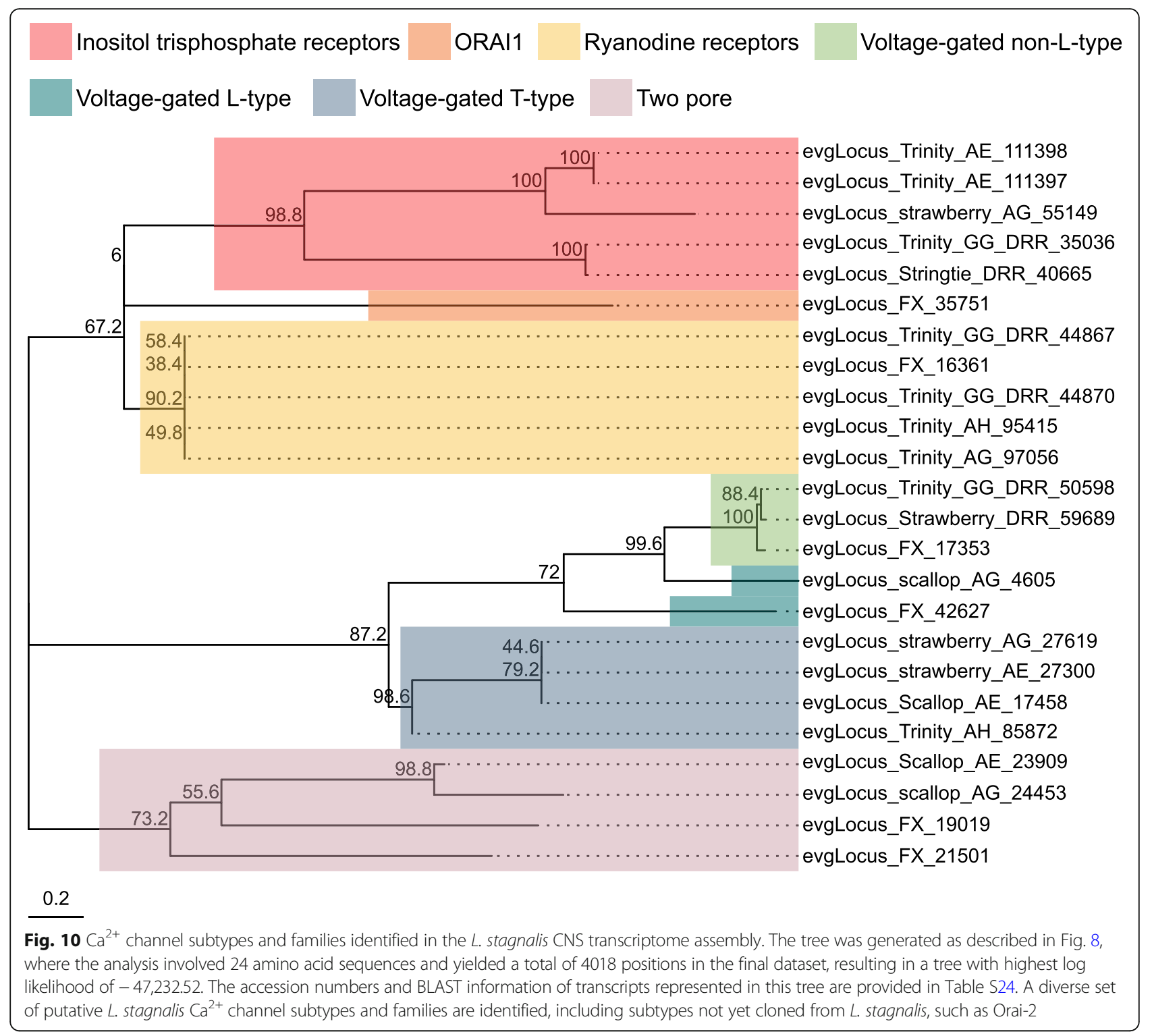

and/or ionotropic neurotransmitter receptors [38, 7577], we examined the protein sequence similarity of each identified $L$. stagnalis channel/receptor transcripts with its homolog in M. musculus, $X$. tropicalis, D. rerio, D. melanogaster, and C. elegans.

Consistent with the importance of intracellular $\mathrm{Ca}^{2+}$ dynamics and homeostasis for neuronal function [78], we found that transcripts encoding the intracellular calcium release channels, ryanodine receptors and inositol triphosphate receptors, and voltage-gated $\mathrm{Ca}^{2+}$ channels in $L$. stagnalis show the highest degrees of sequence similarity with their homologs in the other five species (Fig. 15). The non-selective $\mathrm{Na}^{+}$-leak channel NALCN also exhibited a high degree of sequence conservation between $L$. stagnalis and the other five species, which is consistent with the necessary role of NALCN in regulating basal membrane potential in L. stagnalis [62, 75], M. musculus [79], D. melanogaster [80] and C. elegans neurons [81]. Interestingly, a member of the TRP channel family, transient receptor potential melastatin 1 (TRPM1), also showed a moderate degree of sequence conservation between species. Lower bitscores are consistent with the fact that invertebrate TRPM channels may be divided into broader $\alpha$ - and $\beta$-subfamilies, unlike vertebrates, which express TRPM1-8 $[82,83]$. This relative conservation may point to a conserved function of TRPM1 in phototransduction across organisms, as has been identified in $M$. musculus and D. melanogaster [84], suggesting future investigation may be warranted in studying the role of TRPM1-like proteins in L. stagnalis phototransduction. Meanwhile, the acetylcholine receptor family of ligand-gated ion channels in $L$. stagnalis 


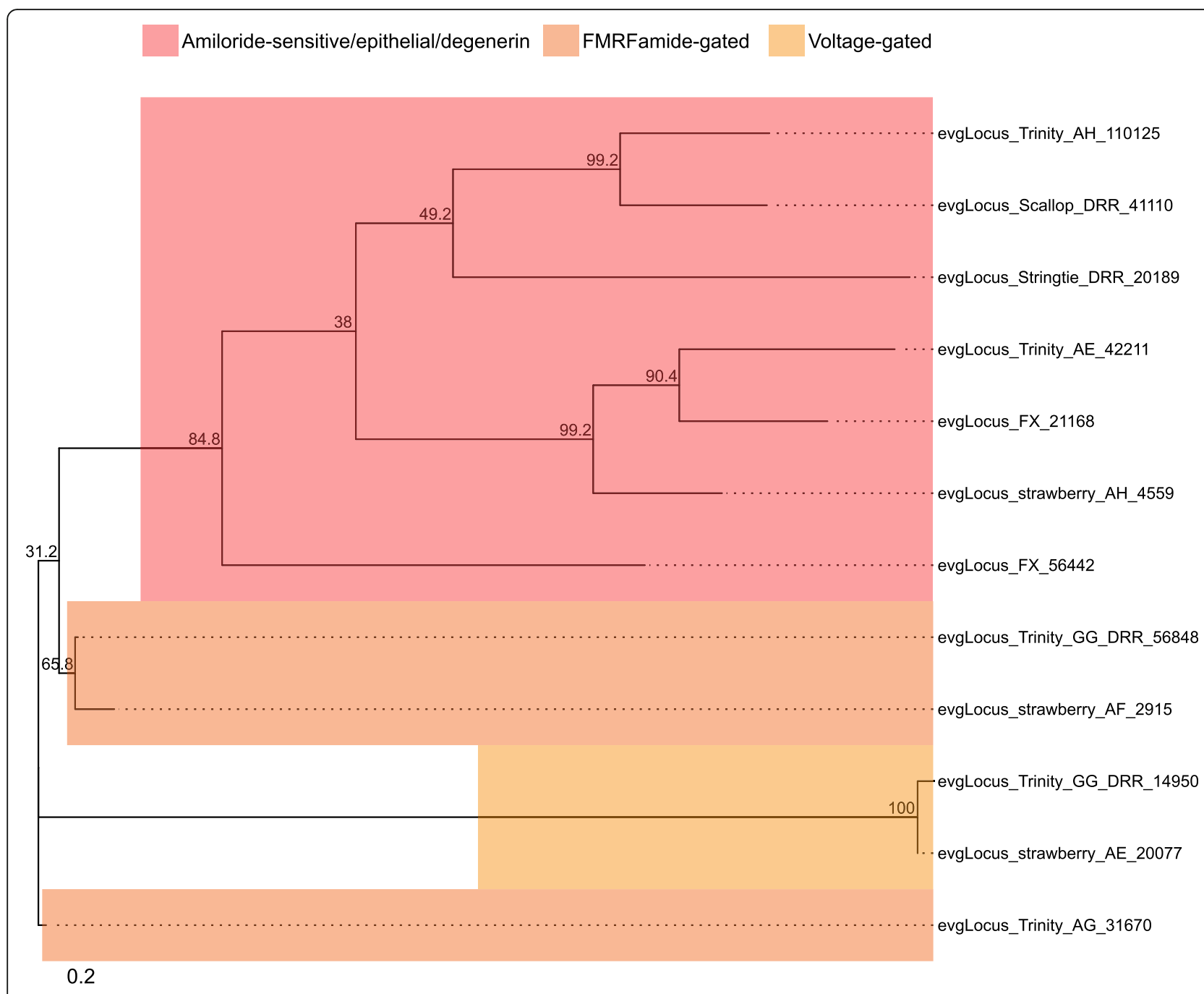

Fig. $11 \mathrm{Na}^{+}$channel subtypes and families identified in the L. stagnalis CNS transcriptome assembly. The tree was generated as described in Fig. 8, where the analysis involved 12 amino acid sequences and yielded a total of 345 positions in the final dataset, resulting in a tree with highest log likelihood of -6272.64 . The accession numbers and BLAST information of transcripts represented in this tree are provided in Table S25. A variety of putative L. stagnalis $\mathrm{Na}^{+}$channel subtypes and families are identified

showed a low degree of sequence similarity with other species. This corresponds well with known expansions in acetylcholine receptor sequences, particularly nicotinic acetylcholine receptors, in molluscs as compared to vertebrate species [85], and consistent with previous studies on L. stagnalis acetylcholine receptors [86]. Taken together, this study provides the resources for future genetic and bioinformatic studies of various ion channels, thus opening up exciting new venues for exploring fundamental and novel mechanisms of neural function in L. stagnalis.

As L. stagnalis is a key model organism used to examine both conserved and novel mechanisms of neurobiology, it is of immense interest to compare and contrast its CNS gene expression with that of other common neuroscience model organisms, in order to better understand the extent to which CNS functions are conserved and consequently how generalizable experimental findings are across species. Here, we compared $L$. stagnalis CNS gene expression with that of $M$. musculus, $X$. tropicalis, D. rerio, D. melanogaster, and C. elegans using several approaches. A comparison of the top 20 most abundantly expressed protein-coding transcripts in these five species (Table 1, Tables S7, S8, S9, S10 and S11) with those in L. stagnalis found several common functional categories critical for CNS function, including energy production, signaling and protein synthesis. Surprisingly, no clear distinctions in terms of prevalent functions were observed between the three vertebrate and three invertebrate species. Comparison of KOG annotations of all the expressed protein-coding transcripts in each species provides a complementary perspective 


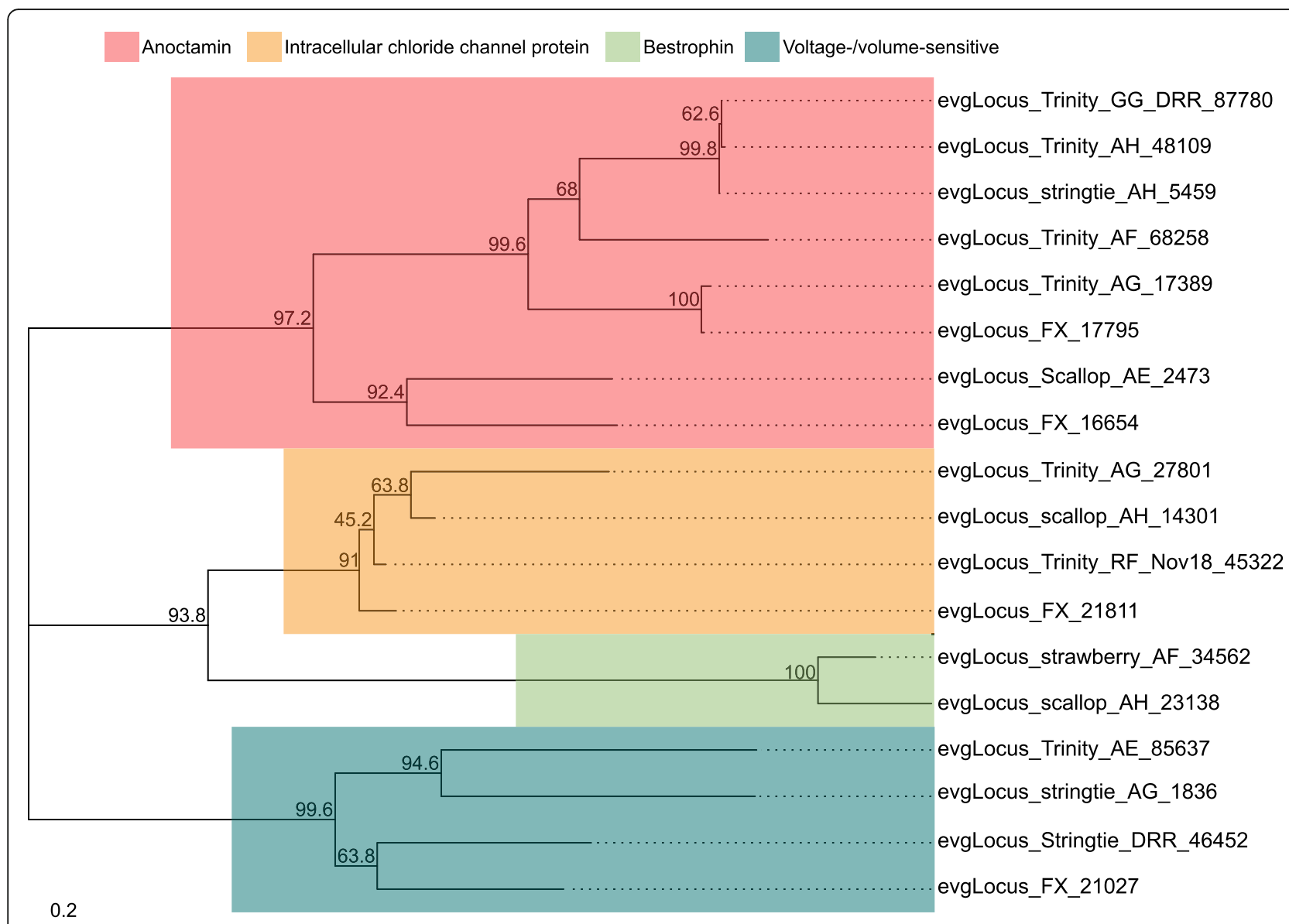

Fig. $12 \mathrm{Cl}^{-}$channel subtypes and families identified in the L. stagnalis CNS transcriptome assembly. The tree was generated as described in Fig. 8, where the analysis involved 18 amino acid sequences and yielded a total of 1233 positions in the final dataset, resulting in a tree with highest log likelihood of $-22,161.37$. The accession numbers and BLAST information of transcripts represented in this tree are provided in Table S26. A variety of putative $L$. stagnalis $\mathrm{Cl}^{-}$channel subtypes and families are identified, of which anoctamin, bestrophin, and intracellular chloride channel protein have not yet been cloned from L. stagnalis

(Fig. 3). While the distribution of transcripts across functional categories was largely similar across the six species, vertebrate species were shown to have more transcripts involved in signal transduction, gene expression and cytoskeleton dynamics, whereas in invertebrates, more transcripts were shown to be related to metabolism and protein synthesis. In the case of cytoskeleton dynamics, this is consistent with known differences in multidirectional polarity and absence of dendritic spines in invertebrate neurons as compared to their vertebrate counterparts [87]. On the other hand, reasons for enrichment of transcripts related to metabolism and protein synthesis in invertebrates are less clear, especially given that metabolic regulation is generally less complex in invertebrates than vertebrates [88]. However, short neuropeptide F signaling, which is not present in vertebrates and plays a role in regulating metabolism and energy homeostasis in invertebrates [89], may account for this difference in metabolism KOG annotations. Subsequently, differences in metabolic pathways between vertebrate and invertebrate CNS may be an interesting avenue of future study.

Finally, we clustered all protein-coding transcripts expressed in the CNS of these six species into orthogroups, each of which was predicted based on sequence similarities to be descended from a common gene in the last common ancestor of these six species. As orthologous genes have been shown to exhibit similar functions [90, 91] and expression patterns [92] across species, such analyses may provide insights into conserved CNS functions across divergent species. Indeed, functional enrichment of genes encoded by transcripts in orthogroups shared amongst all six species identified processes fundamental to CNS functioning, such as glutamate receptor signaling, ion transport, and mitochondrial aerobic respiration (Fig. 5). Conversely, analyses of orthogroups shared amongst only the vertebrate (Fig. 6) or invertebrate (Fig. 7) species examined showed that 


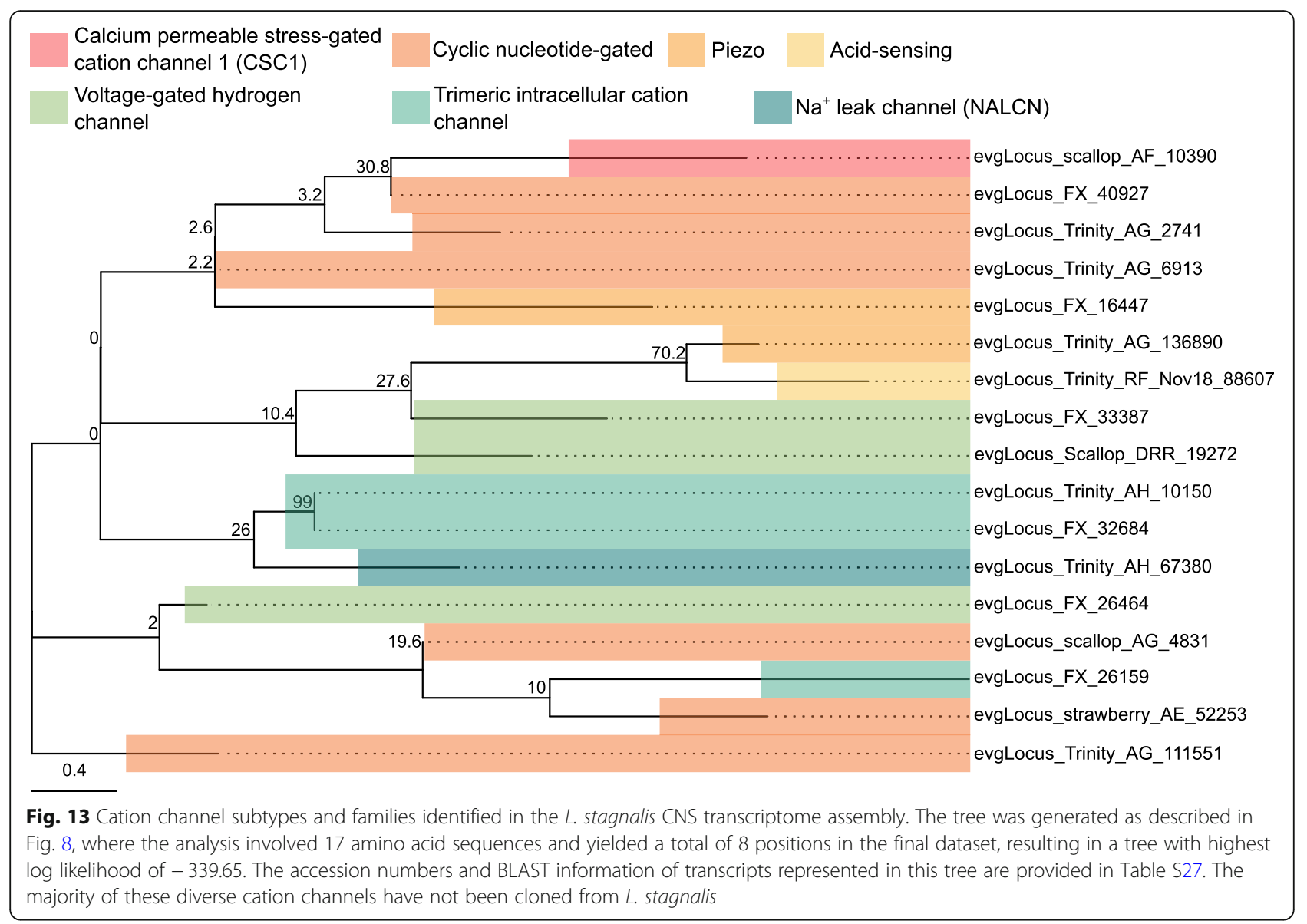

functions related to the immune/inflammatory response, actin dynamics and $\mathrm{G}$ protein-coupled receptor (GPCR) signaling were likely less conserved between the two groups. This is expected, as invertebrates lack acquired immune responses and thus the genes associated with acquired immunity, relying entirely on innate immunity to defend against infection [93, 94]. Similarly, most invertebrates lack dendritic spines, plasticity of which relies on actin remodeling [95], and differential conservation of actin dynamics between vertebrates and invertebrates identified in the current study is consistent with this. Finally, differences in enrichment of genes related to GPCR signaling is expected due to differences in the ensemble of GPCRs expressed in invertebrates compared to vertebrates. For instance, $C$. elegans expresses nematode chemosensory (NCHM) GPCRs, which are not present in vertebrates [96]. Additionally, some invertebrates lack various $\mathrm{G} \alpha$ proteins, including Gai/o and Goq subtypes [97]. A full characterization of GPCRs in L. stagnalis may be thus of interest for future study.

\section{Conclusion}

We have shown that L. stagnalis had more orthogroups in common with the three vertebrate species ( $M$. musculus, D. rerio, and $X$. tropicalis) than D. melanogaster or C. elegans, suggesting that it may be an advantageous system in which to leverage the simplicity of an invertebrate CNS but also maintain more conserved molecular mechanisms with higher organisms. To the best of our knowledge, there have been few systematic comparisons of CNS physiology in commonly used vertebrate and invertebrate model organisms. Such comparisons can provide insights into the evolutionary history of various cellular processes and protein families. Our findings in this study provide a starting point for better understanding of the functional commonalities and specializations of the vertebrate and invertebrate CNS, which is critically needed to inform future studies in terms of the suitability of the chosen model and generalizability of future findings.

\section{Methods}

\section{RNA-seq and pre-processing of reads}

The central ganglia ring from four adult L. stagnalis were dissected according to established protocols [49, $63,98,99]$ and total RNA was extracted using the Trizol method as previously described [7]. RNA quality was evaluated by capillary electrophoresis migration on an 


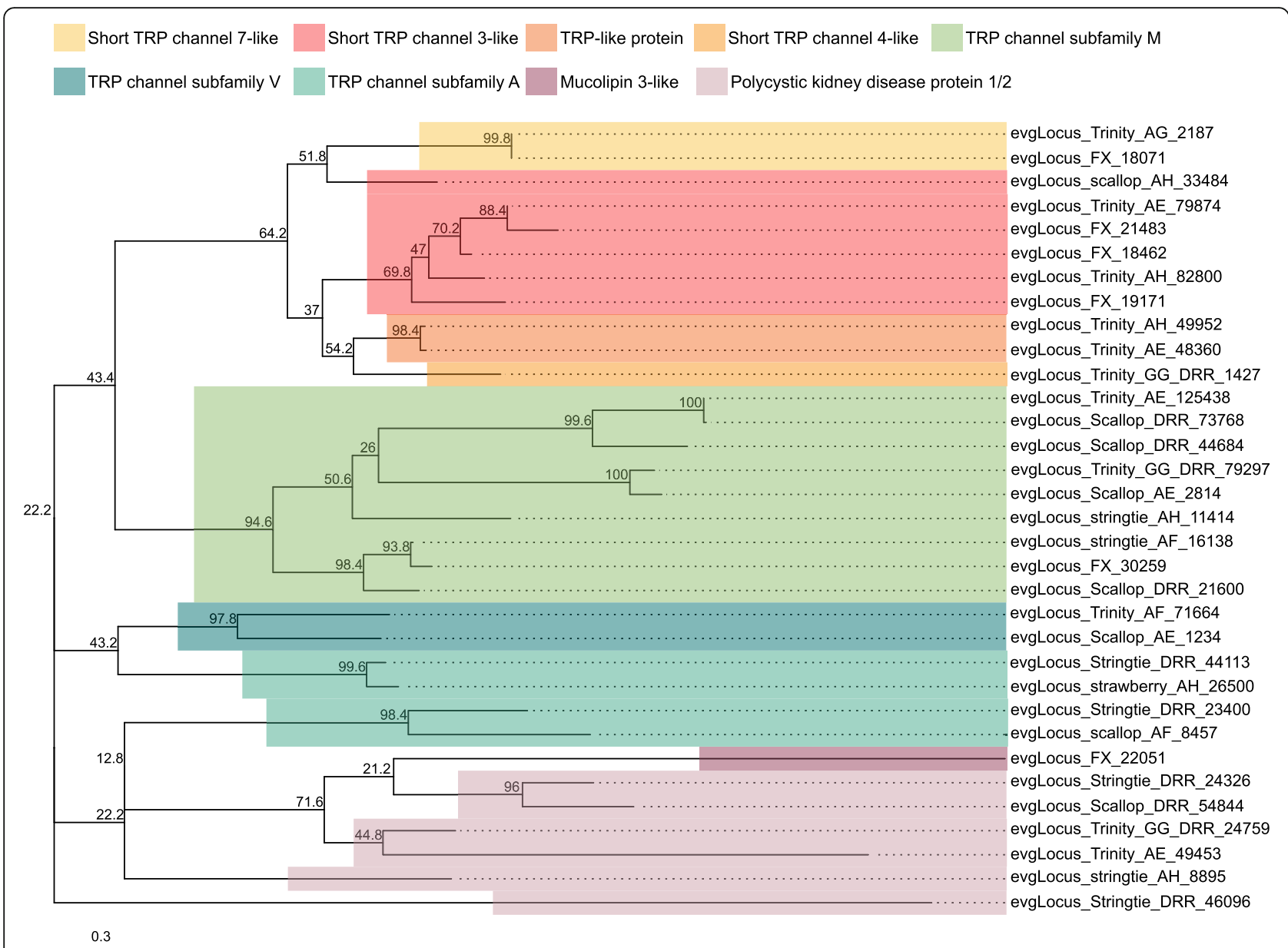

Fig. 14 Transient receptor potential (TRP) channel subtypes and families identified in the L. stagnalis CNS transcriptome assembly. The tree was generated as described in Fig. 8, where the analysis involved 33 amino acid sequences and yielded a total of 133 positions in the final dataset, resulting in a tree with highest log likelihood of -7343.03 . The accession numbers and BLAST information of transcripts represented in this tree are provided in Table S28. This suggests a vertebrate-like diversity of TRP channels expressed by L. stagnalis

Agilent Bioanalyzer, using the Agilent RNA Pico 6000 LabChip kit. The RNA integrity number (RIN) score was not able to be calculated due to the lack of $28 \mathrm{~S}$ RNAassociated peak on electropherograms of L. stagnalis RNA. This phenomenon occurs due to a thermolabile hidden break in the molecule, as described in various invertebrates, which leads to its cleavage into two subparts of size similar to that of 18S RNA [100]. For this reason, RNA quality was evaluated using the global shape of migration profiles. One $150 \mathrm{bp}$ paired-end RNA-seq library was prepared from $500 \mu \mathrm{g}$ of total RNA from each central ring ganglia sample, using the TruSeq Stranded mRNA kit (Illumina, San Diego, CA, USA). Each library was sequenced using 151-bp paired-end reads chemistry on a HiSeq 4000 Illumina sequencer, where 20 to 22 million reads were generated per library and all 4 libraries were sequenced on a single lane. The raw data were filtered to remove any clusters that had too high intensity corresponding to bases other than the called base. By default, the purity of the signal from each cluster was examined over the first 25 cycles and calculated as Chastity = Highest_Intensity / (Highest_Intensity + Next_Highest_Intensity) for each cycle. The default filtering implemented at the base calling stage allows at most one cycle that is less than the Chastity threshold (0.6). The reads were further processed by trimming, using the fastx clean script from the FASTX toolkit [101]: 1) adapters and primers on the whole read, 2) low quality nucleotides from both ends (where quality value was lower than 20), and 3) sequences between the second unknown nucleotide $(\mathrm{N})$ and the end of the read. Finally, reads that either 1) were shorter than 30 nucleotides, 2) originated from the lowconcentration spike-in library of Illumina PhiX Control or 3) corresponded to ribosomal RNA, as filtered by SortMeRNA v 1.0 [102] were removed. 


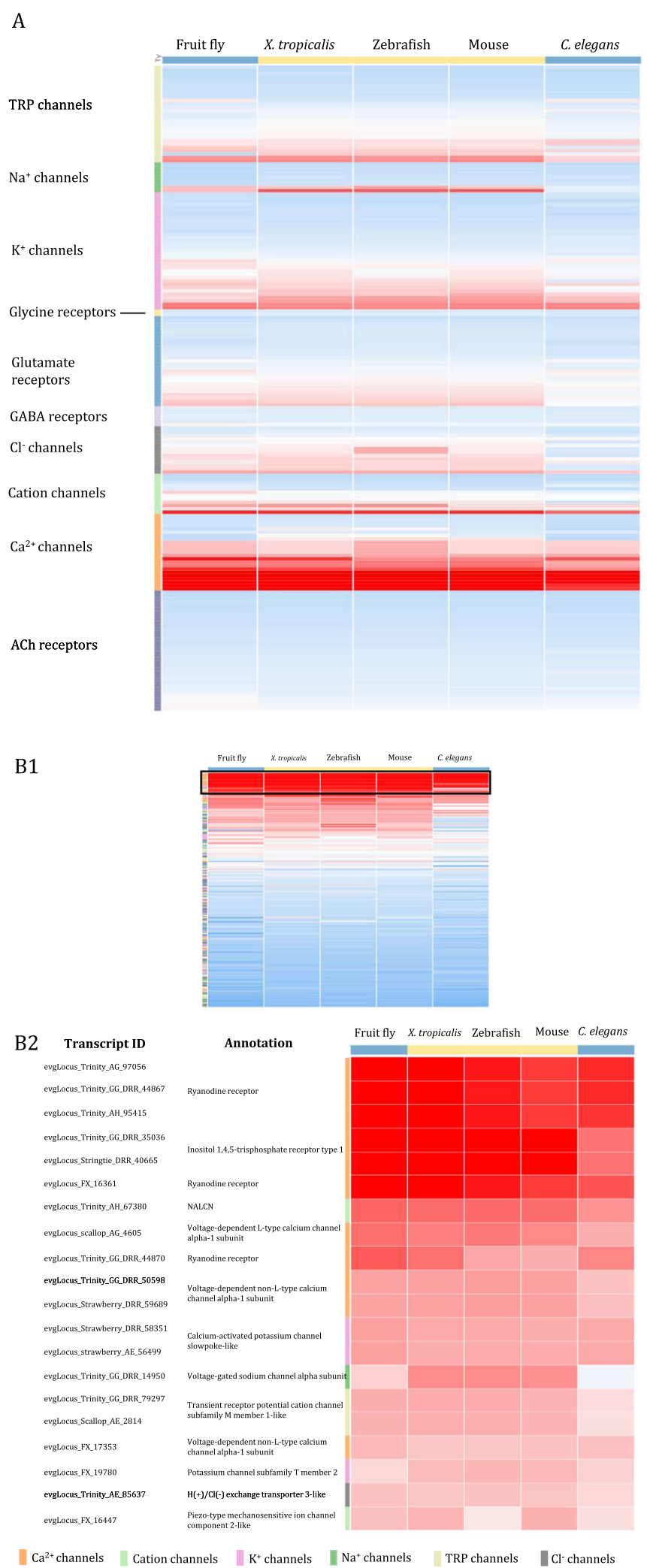

Fig. 15 (See legend on next page.) 
(See figure on previous page.)

Fig. 15 Heat maps of protein sequence similarity as measured by BLASTP bitscore between putative L. stagnalis ion channel/ionotropic receptor transcripts and homologs in mouse, X. tropicalis, zebrafish, fruit fly and C. elegans. Each bitscore is standardized by the average and standard deviation of all bitscores in the matrix. Each row corresponds to a single L. stagnalis transcript and each column to a species, such that each cell represents a standardized bitscore. Colour of each cell is proportional to the bitscore, where a darker colour indicates higher bitscore and consequently sequence similarity. A, Transcripts are sorted by the ion channel/ionotropic receptor class and species are sorted in descending order by the absolute sum of bitscores of its homologs, i.e. species with the highest bitscores across all transcripts is on the left of the heatmap. Transcripts encoding $\mathrm{Ca}^{2+}$ and cation channels appear to share the greatest sequence similarity across all six species. B1 Transcripts are sorted in descending order by the absolute sum of bitscores, i.e. transcripts with the highest sequence similarities across species are at the top of the heatmap. B2 The top 20 transcripts thus ranked are labeled by transcript ID and annotation. The colour scale is adjusted as compared to in B1 to increase contrast between cells. Interactive Clustergrammer heat map can be accessed at http://amp.pharm.mssm.edu/clustergrammer/viz/5b6 ba1ca7226c37ceda3505b/LS_channel_comparisons.txt. The majority of these top 20 transcripts encode calcium channels

\section{Transcriptome assembly and annotation of predicted protein-coding transcripts}

Workflow for assembling and identifying protein-coding transcripts in the L. stagnalis CNS transcriptome is summarized in Fig. 1. Read quality was visualized using FastQC [103], and then analyzed using rCorrector [104], which employs a k-mer based method to correct random sequencing errors in Illumina RNA-seq reads. Reads were mapped to the genome assembly using STAR [105] and assembled using Stringtie [106], Scallop [107], Strawberry [108], and Trinity [109] genome-guided assembly programs, as well as Trinity de novo assembly program. This resulted in four genome-guided assemblies per sample (16), one de novo assembly per sample (4). Reads that were flagged as erroneous but unfixable were removed using a custom script (https://github. com/harvardinformatics/TranscriptomeAssemblyTools/ blob/master/FilterUncorrectabledPEfastq.py), and processed and assembled in the same manner, except using a modified custom script to remove unfixable single-end reads after rCorrector analysis. This resulted in five additional assemblies: four genomeguided assemblies and one de novo assembly of unmapped reads. These 22 assemblies (including four per sample from each assembly program, one de novo unmapped assembly, and the above additional assemblies) were merged and processed through the EvidentialGene tr2aacds pipeline [110] to identify a set of non-redundant potential protein-coding transcripts, as previously described $[111,112]$. This pipeline removes perfect duplicate sequences, identifies and clusters highly similar sequences through a "all-vs-all BLAST" (98\% similarity by default) process, and identifies potential open reading frames (ORFs) in each transcript. The final set of predicted ORF-containing transcripts (>100 bp) were sorted into three sets: "okay" for primary transcripts, "okalt" for alternative transcripts and "drops" for redundant, fragmented or too short transcripts. Transcripts containing complete or 3'-partial open ORFs were extracted from the primary and alternate sets for downstream analyses. Expression levels of the transcripts were assessed using Salmon [113] to allow for expression-based filtering of potential transcript artifacts (TPM $>0$ in all five RNA-seq libraries). The resultant transcripts were searched against the Pfam database and analyzed using the signaling peptide identification programs SignalP and Phobius to assess their potential as protein-coding transcripts. All predicted protein coding sequences were annotated against the Swissprot, $\mathrm{Nr}$, KOG databases and the L. stagnalis predicted gene set (E-value cut-off of 1E-5). BUSCO analysis [114] was performed to assess the completeness of this set of transcripts (E-value cut-off of 1E-5). The Salmon software was used to calculate the expression level of the predicted coding transcripts in each of the four paired-end reads libraries. The transcripts were ranked by their expression level in each of the four libraries sequenced for this study, and the median rank for each transcript was calculated across all four libraries to identify the top 20 expressed transcripts.

\section{Comparison with previously published $L$. stagnalis CNS transcriptome libraries and aggregation of protein-coding sequence library}

For comparison with the current assembly, the previously published EST library [49] and de novo assembled transcripts [50] from the L. stagnalis CNS were obtained from the European Nucleotide Archive and processed in the same manner as described above to predict protein-coding sequences. The amino acid sequences were annotated against $\mathrm{Nr}$ database using BLASTP (E-value cut-off of 1E-5). Finally, the amino acid sequences of predicted protein-coding sequences from these two libraries and the current assembly were clustered and deduplicated (100\% similarity cutoff) separately using CD-HIT [115]. The resultant set of sequences was analyzed by BUSCO to assess completeness (E-value cut-off of $1 \mathrm{E}-5)$. 
Analyses of published CNS RNA-seq libraries from representative model organisms of animals

Published RNA-seq libraries of M. musculus [53], D. rerio [54], $X$. tropicalis [53], D. melanogaster (PRJNA320764), and C. elegans [55] CNS were obtained from the NCBI Short Reads Archive (Table S1). Where possible, equal numbers of sequencing libraries from both male and female animals were analyzed. Quality of reads was visualized using FastQC, and erroneous bases were corrected and filtered in the same manner as described above. The fastp program [116] was then employed to remove adapters and trim bases with phred score $<5$, as previously recommended [117]. The software Salmon [113] was used to map reads to reference transcripts (both cDNAs and non-coding RNAs) for each species. Protein sequences of transcripts with TPM $>0$ in all libraries examined for the species were collected for all species examined and clustered using Orthofinder [118] to identify orthogroups. Membership of each species in orthogroups were visualized using UpSetR [119]. Gene IDs of protein-coding transcripts with TPM $>0$ in all libraries of each species were retrieved from Biomart via the biomaRt $\mathrm{R}$ package [120] for functional enrichments using g: Profiler R package [121], where IEA were excluded and statistical significance was calculated using the gSC method (p-adjusted $<0.05$ cut-off). Clustering of Gene Ontology (GO) terms was visualized using REViGO [122].

\section{Identification of transcripts encoding ion channels and ionotropic receptors and interspecies comparisons}

Pfam IDs containing the word "channel" in their descriptions were obtained from Pfam (pfam.xfam.org) (Table S2). Predicted protein-coding transcripts containing at least one of this set of protein domains were then analyzed using Phobius and TMHMM, and only those predicted to contain transmembrane helices by both programs were retained. The putative ion channel/receptor sequences were annotated against the $\mathrm{Nr}$ database (E-value cut-off of $1 \mathrm{E}-5$ ) and manually inspected to separate into ionotropic neurotransmitter receptor and $\mathrm{Na}^{+}, \mathrm{K}^{+}, \mathrm{Ca}^{2+}, \mathrm{Cl}^{-}$, and cation channel subfamilies. The sequences within each subfamily were aligned and clustered using T-Coffee [123], trimmed using trimAl [124], and phylogenetic trees were computed using the Maximum Likelihood method and JTT matrix-based model [125] in MEGA $X$ [126]. Initial tree(s) for the heuristic search were obtained automatically by applying Neighbor-Join and BioNJ algorithms to a matrix of pairwise distances estimated using the JTT model, and then selecting the topology with superior log likelihood value. Phylogenetic trees were visualized using the ggtree package for R [127].M. musculus, X. tropicalis, D. rerio, D. melanogasterand $C$. elegans homologs of the putative $L$. stagnalis ion channel/receptor transcripts were identified via BLASTP (E-value cut-off of 1E-5). The heatmap of standardized BLAST bitscores was visualized using Clustergrammer [128].

\section{Supplementary Information}

The online version contains supplementary material available at https://doi. org/10.1186/s12864-020-07287-2.

\begin{abstract}
Additional file 1: Table S1. Published adult CNS RNA-seq libraries from selected model organisms used in this study. Table S2. Pfam IDs used to identify transcripts encoding ion channels and ionotropic receptors in the L. stagnalis CNS. Table S3. Summary of L. stagnalis CNS RNA-seq library metrics before and after reads correction and filtering. Table S4. Mapping statistics of the CNS RNA-seq libraries to L. stagnalis genome assembly. Table S5. Transcript assembly statistics for L. stagnalis CNS RNAseq libraries. Table S6. Proportion of transcripts containing complete and fragmented ORFs as identified by the Evigene pipeline in the "okay" and "okalt" sequence sets. Table S7. Top 20 expressed transcripts in the adult mouse brain. Table S8. Top 20 expressed transcripts in the adult $X$. tropicalis brain. Table S9. Top 20 expressed transcripts in the adult zebrafish brain. Table S10. Top 20 expressed transcripts in the adult fruitfly brain. Table S11. Top 20 expressed transcripts in adult C. elegans neurons. Table S12. Enriched GO terms of mouse genes in orthogroups shared amongst vertebrate and invertebrate species. Table S13. Enriched Reactome pathways of mouse genes in orthogroups shared amongst all the species examined. Table S14. Enriched KEGG pathways of mouse genes in orthogroups shared amongst all the species examined. Table S15. Transcript factors whose binding motifs are enriched in the set of mouse genes in orthogroups shared amongst all the species examined. Table S16. Enriched GO terms of mouse genes in orthgroups shared amongst only the vertebrate species examined. Table S17. Enriched Reactome pathways of mouse genes in orthgroups shared amongst only the vertebrate species examined. Table S18. Enriched KEGG pathways of mouse genes in orthgroups shared amongst only the vertebrate species examined. Table S19. Enriched GO terms of fruit fly genes in orthgroups shared amongst only the invertebrate species examined.

Additional file 2: Table S20. Transcripts encoding ionotropic acetylcholine receptors in the L. stagnalis CNS. Table S21. Transcripts encoding ionotropic GABA or glycine receptors in the L. stagnalis CNS. Table S22. Transcripts encoding ionotropic glutamate receptors in the $L$. stagnalis CNS. Table S23. Transcripts encoding K+ channels in the $L$. stagnalis CNS. Table S24. Transcripts encoding Ca2+ channels in the $L$. stagnalis CNS. Table S25. Transcripts encoding Na + channels in the $L$. stagnalis CNS. Table S26. Transcripts encoding Cl- channels in the $L$. stagnalis CNS. Table S27. Transcripts encoding cation channels in the $L$. stagnalis CNS. Table S28. Transcripts encoding TRP channels in the $L$. stagnalis CNS.
\end{abstract}

\section{Abbreviations}

AA: Amino acid; BLAST: Basic local alignment search tool; CNS: Central nervous system; CPG: Central pattern generator; CRAC: Calcium releaseactivated current; EST: Expressed sequence tag; GABA: Gamma-aminobutyric acid; GO: Gene ontology; GPCR: G protein-coupled receptor;

HCN: Hyperpolarization-activated cyclic nucleotide-gated; KEGG: Kyoto Encyclopedia of Genes and Genomes; KOG: Eukaryotic orthologous group; NALCN: Sodium $\left(\mathrm{Na}^{+}\right)$leak channel non-selective; ORF: Open reading frame; RIN: RNA integrity number; RNA: Ribonucleic acid; RyR: Ryanodine receptors; TPM: Transcripts per kilobase million; TRP: Transient receptor potential 


\section{Authors' contributions}

$\mathrm{ND}$, JB, and ZPF interpreted the data. ND and JB performed bioinformatic analysis and drafted the manuscript. ZZ and YW participated in the bioinformatic analysis. ND and ZPF carried out animal dissections and prepared the tissue samples. KL and BN conducted RNA-sequencing and quality control. AD, JMK, MAC, and ZPF initiated the sequencing project. ZZ, HSS, and ZPF initiated the bioinformatic project, and ND and ZPF designed the project. JB, ZZ, YW, JMK, HSS, MAC, and ZPF participated in producing the final version of the manuscript. HSS, MAC, and ZPF conceptualized the study. All authors read and approved the final manuscript.

\section{Funding}

This work was supported by grants from the National Sciences and Engineering Research Council of Canada to ZPF (RGPIN-2014-0671) and HSS (RGPIN-2016-04574), and by the Genoscope, the Commissariat à l'Energie Atomique et aux Energies Alternatives (CEA) and a grant from France Génomique (ANR-10-INBS-09-08) to MAC for the Programme STAGING. ND was a recipient of the NSERC Post-Graduate Scholarship, Doctoral (NSERCCGS-D). JB was a recipient of the Ontario Graduate Scholarship (OGS) and NSERC Post-Graduate Scholarship, Doctoral (NSERC-PGS-D).

\section{Availability of data and materials}

The raw sequence reads have been deposited in the Sequence Read Archive (SRA) under accession numbers ERX4742139-ERX4742142, and all associated project data are available under the study identifier PRJEB41522 at NCBI or EBI (as well as http://www.lymnaea.org/)

\section{Ethics approval and consent to participate}

All experiments were performed according to the guidelines of the Animal Care Committee of the University of Toronto.

\section{Consent for publication}

Not applicable.

\section{Competing interests}

The authors declare that they have no competing interests.

\section{Author details}

'Department of Physiology, University of Toronto, 3308 MSB, 1 King's College Circle, Toronto, ON M5S 1A8, Canada. ${ }^{2}$ Donnelly Centre for Cellular and Biomolecular Research and Department of Molecular Genetics, University of Toronto, Toronto, ON M5S 3E1, Canada. ${ }^{3}$ Department of Ecology and Evolutionary Biology, University of Toronto, Toronto, Ontario M5S 3B2, Canada. ${ }^{4}$ Department of Biological Sciences, University of Toronto Scarborough, Toronto, Ontario M1C 1A4, Canada. ${ }^{5}$ Genoscope, Institut de biologie François Jacob, Commissariat à l'Energie Atomique (CEA), Université Paris-Saclay, BP5706, 91057 Evry, France. ${ }^{6}$ Génomique Métabolique, Genoscope, Institut François Jacob, CEA, CNRS, University of Evry, Université Paris-Saclay, 91057 Evry, France. 'School of Life Sciences, University of Nottingham, University Park, Nottingham, UK NG7 2RD, UK. ${ }^{8}$ Department of Ecological Science, Faculty of Science, Vrije Universiteit, Amsterdam, The Netherlands. ${ }^{9}$ Department of Surgery, University of Toronto, Toronto, Ontario M5S 1A8, Canada. ${ }^{10}$ ESE, Ecology and Ecosystem Health, INRAE, Agrocampus Ouest, 35042 Rennes, France.

Received: 4 June 2020 Accepted: 28 November 2020

Published online: 06 January 2021

\section{References}

1. Spencer GE, Kazmi MH, Syed NI, Lukowiak K. Changes in the activity of a $\mathrm{CpG}$ neuron after the reinforcement of an operantly conditioned behavior in Lymnaea. J Neurophysiol. 2002;88(4):1915-23.

2. Scheibenstock A, Krygier D, Haque Z, Syed N, Lukowiak K. The soma of RPeD1 must be present for long-term memory formation of associative learning in Lymnaea. J Neurophysiol. 2002;88(4):1584-91.

3. Sunada H, Watanabe T, Hatakeyama D, Lee S, Forest J, Sakakibara M, Ito E, Lukowiak K. Pharmacological effects of cannabinoids on learning and memory in Lymnaea. J Exp Biol. 2017:220(17):3026-38.

4. Syed N, Bulloch A, Lukowiak K. In vitro reconstruction of the respiratory central pattern generator of the mollusk Lymnaea. Science. 1990 250(4978):282-5.
5. Elliott $\mathrm{CJH}$, Vehovszky Á. Polycyclic neuromodulation of the feeding rhythm of the pond snail Lymnaea stagnalis by the intrinsic octopaminergic interneuron, OC. Brain Res. 2000;887(1):63-9.

6. Cheung U, Moghaddasi M, Hall HL, Smith JJB, Buck LT, Woodin MA. Excitatory actions of GABA mediate severe-hypoxia-induced depression of neuronal activity in the pond snail (Lymnaea stagnalis). J Exp Biol. 2006; 209(22):4429-35.

7. Nejatbakhsh N, Guo CH, Lu TZ, Pei L, Smit AB, Sun HS, van Kesteren RE, Feng ZP. Caltubin, a novel molluscan tubulin-interacting protein, promotes axonal growth and attenuates axonal degeneration of rodent neurons. J Neurosci. 2011;31(43):15231-44

8. OECD: Test No. 243: Lymnaea stagnalis Reproduction Test; 2016.

9. Niyogi S, Brix KV, Grosell M. Effects of chronic waterborne nickel exposure on growth, ion homeostasis, acid-base balance, and nickel uptake in the freshwater pulmonate snail, Lymnaea stagnalis. Aquat Toxicol. 2014;150:36-44

10. Crémazy A, Brix KV, Wood CM. Chronic toxicity of binary mixtures of six metals ( $\mathrm{Ag}, \mathrm{Cd}, \mathrm{Cu}, \mathrm{Ni}, \mathrm{Pb}$, and $\mathrm{Zn}$ ) to the great pond snail Lymnaea stagnalis. Environ Sci Technol. 2018:52(10):5979-88.

11. Mazur R, Shubiao W, Szoszkiewicz K, Bedla D, Nowak A, Mazur R, Shubiao W, Szoszkiewicz K, Bedla D, Nowak A. A Lymnaea stagnalis embryo test for toxicity bioindication of acidification and ammonia pollution in water. Water. 2016;8(7):295

12. Bouétard A, Noirot C, Besnard A-L, Bouchez O, Choisne D, Robe E, Klopp C, Lagadic L, Coutellec M-A. Pyrosequencing-based transcriptomic resources in the pond snail Lymnaea stagnalis, with a focus on genes involved in molecular response to diquat-induced stress. Ecotoxicology. 2012;21(8): 2222-34.

13. Bouétard A, Besnard A-L, Vassaux D, Lagadic L, Coutellec M-A. Impact of the redox-cycling herbicide diquat on transcript expression and antioxidant enzymatic activities of the freshwater snail Lymnaea stagnalis. Aquat Toxicol. 2013:126:256-65.

14. Akande IS, Odetola AA. In: El Ridi R, editor. Epidemiological Survey of Human and Veterinary Schistosomiasis. London: InTech; 2013.

15. Wang T, Zhao M, Liang D, Bose U, Kaur S, McManus DP, Cummins SF. Changes in the neuropeptide content of Biomphalaria ganglia nervous system following Schistosoma infection. Parasit Vectors. 2017;10(1):275.

16. Adema CM, Hillier LW, Jones CS, Loker ES, Knight M, Minx P, Oliveira G, Raghavan N, Shedlock A, do Amaral LR, et al. Whole genome analysis of a schistosomiasis-transmitting freshwater snail. Nat Commun. 2017;8:15451.

17. Catterall WA, Dib-Hajj S, Meisler MH, Pietrobon D. Inherited neuronal ion Channelopathies: new windows on complex neurological diseases. J Neurosci. 2008:28(46):11768-77.

18. Hubner CA, Jentsch TJ. Ion channel diseases. Hum Mol Genet. 2002;11(20): $2435-45$.

19. Rudy B. Diversity and ubiquity of K channels. Neuroscience. 1988;25(3):72949

20. Coetzee WA, Amarillo Y, Chiu J, Chow A, Ozaita A, Pountney D, Saganich M, Vega-Saenz de Miera E, Rudy B. Molecular diversity of K+ channels. Ann NY Acad Sci. 1999;868:233-85.

21. Maljevic S, Lerche H. Potassium channels: a review of broadening therapeutic possibilities for neurological diseases. J Neurol. 2013;260(9): 2201-11.

22. De Waard M, Gurnett CA, Campbell KP. In: Narahashi T, editor. Structural and functional diversity of voltage-activated calcium channels. New York: Plenum Press; 1996. p. 41-87.

23. Goldin AL. Diversity of mammalian voltage-gated sodium channels. Ann N Y Acad Sci. 1999:868:38-50

24. Betz H. Ligand-gated ion channels in the brain: the amino acid receptor superfamily. Neuron. 1990;5(4):383-92.

25. Traynelis SF, Wollmuth LP, McBain CJ, Menniti FS, Vance KM, Ogden KK, Hansen KB, Yuan H, Myers SJ, Dingledine R. Glutamate receptor ion channels: structure, regulation, and function. Pharmacol Rev. 2010;62(3):405-96.

26. Sigel E, Steinmann ME. Structure, function, and modulation of GABA receptors. J Biol Chem. 2012;287(48):40224-31.

27. Wu J, Lukas RJ. Naturally-expressed nicotinic acetylcholine receptor subtypes. Biochem Pharmacol. 2011;82(8):800-7.

28. Barnes NM, Sharp T. A review of central 5-HT receptors and their function. Neuropharmacology. 1999;38(8):1083-152.

29. Burnstock G, Kennedy C. P2X receptors in health and disease. Adv Pharmacol. 2011;61:333-72. 
30. Baumann A, Grupe A, Ackermann A, Pongs O. Structure of the voltagedependent potassium channel is highly conserved from Drosophila to vertebrate central nervous systems. EMBO J. 1988;7(8):2457-63.

31. Salkoff $L$, Baker K, Butler A, Covarrubias M, Pak MD, Wei A. An essential 'set' of K+ channels conserved in flies, mice and humans. Trends Neurosci. 1992; 15(5):161-6.

32. Fleishman SJ, Yifrach O, Ben-Tal N. An evolutionarily conserved network of amino acids mediates gating in voltage-dependent potassium channels. J Mol Biol. 2004;340(2):307-18.

33. Harvey RJ, Vreugdenhil E, Barnard EA, Darlison MG. Cloning of genomic and CDNA sequences encoding an invertebrate $\gamma$-aminobutyric acid a receptor subunit. Biochem Soc Trans. 1990;18(3):438-9.

34. Hutton ML, Harvey RJ, Barnard EA, Darlison MG. Cloning of a CDNA that encodes an invertebrate glutamate subunit. FEBS J. 1991;292(1):111-4.

35. Stühmer T, Amar M, Harvey RJ, Bermudez I, Van Minnen J, Darlison MG, Minnen JV, Darlison MG. Structure and pharmacological properties of a molluscan glutamate-gated cation channel and its likely role in feeding behavior. J Neurosci. 1996;16(9):2869-80.

36. Ha TJ, Kohn AB, Bobkova Y, Moroz LL. Molecular characterization of NMDA-like receptors in Aplysia and Lymnaea: relevance to memory mechanisms. Biol Bull. 2006;210(3):255-70.

37. Smit AB, Syed NI, Schaap D, Van Minnen J, Klumperman J, Kits KS, Lodder H, Van Der Schors RC, Van Elk R, Sorgedrager B, et al. A glia-derived acetylcholine-binding protein that modulates synaptic transmission. Nature. 2001;411(6835):261-8.

38. Spafford JD, Chen L, Feng ZP, Smit AB, Zamponi GW. Expression and modulation of an invertebrate presynaptic calcium channel a1 subunit homolog. J Biol Chem. 2003;278(23):21178-87.

39. Spafford JD, Munno DW, Van Nierop P, Feng ZP, Jarvis SE, Gallin WJ, Smit $A B$, Zamponi GW, Syed NI. Calcium channel structural determinants of synaptic transmission between identified invertebrate neurons. J Biol Chem. 2003:278(6):4258-67.

40. Spafford JD, Dunn T, Smit AB, Syed NI, Zamponi GW. In vitro characterization of L-type calcium channels and their contribution to firing behavior in invertebrate respiratory neurons. J Neurophysiol. 2006;95(1):42-52.

41. Senatore A, Spafford JD. Transient and big are key features of an invertebrate T-type channel (LCav3) from the central nervous system of Lymnaea stagnalis. J Biol Chem. 2010;285(10):7447-58.

42. Bavan S, Straub VA, Webb TE, Ennion SJ. Cloning and Characterization of a P2X Receptor Expressed in the Central Nervous System of Lymnaea stagnalis. PLoS One. 2012;7(11):e50487.

43. Adams DJ, Smith SJ, Thompson SH. Ionic currents in Molluscan Soma. Annu Rev Neurosci. 1980;3(1):141-67.

44. Moroz LL, Edwards JR, Puthanveettil SV, Kohn AB, Ha T, Heyland A, Knudsen B, Sahni A, Yu F, Liu L, et al. Neuronal transcriptome of Aplysia: neuronal compartments and circuitry. Cell. 2006;127(7):1453-67.

45. Kenny NJ, Truchado-García M, Grande C. Deep, multi-stage transcriptome of the schistosomiasis vector Biomphalaria glabrata provides platform for understanding molluscan disease-related pathways. BMC Infect Dis. 2016;16(1):618.

46. Gauss R, Seifert R, Kaupp UB. Molecular identification of a hyperpolarizationactivated channel in sea urchin sperm. Nature. 1998:395:583-7.

47. Feske S, Gwack Y, Prakriya M, Srikanth S, Puppel SH, Tanasa B, Hogan PG, Lewis RS, Daly M, Rao A. A mutation in Orai1 causes immune deficiency by abrogating CRAC channel function. Nature. 2006;441(7090):179-85.

48. Prakriya M, Feske S, Gwack Y, Srikanth S, Rao A, Hogan PG. Orai1 is an essential pore subunit of the CRAC channel. Nature. 2006:443(7108):230-3.

49. Feng ZP, Zhang Z, van Kesteren RE, Straub VA, van Nierop $P$, Jin $K$, Nejatbakhsh N, Goldberg Jl, Spencer GE, Yeoman MS, et al. Transcriptome analysis of the central nervous system of the mollusc Lymnaea stagnalis. BMC Genomics. 2009;10:451.

50. Sadamoto H, Takahashi H, Okada T, Kenmoku H, Toyota M, Asakawa Y. de novo sequencing and transcriptome analysis of the central nervous system of mollusc Lymnaea stagnalis by deep RNA sequencing. PLoS One. 2012; 7(8):e42546.

51. Coutellec MA, et al. Reference genome of the freshwater snail Lymnaea stagnalis, a multidisciplinary invertebrate model: overall rationale of the STAG ING project. In: Pearls of wisdom: synergising leadership and expertise in molluscan genomics, a Theo Murphy international scientific meeting by the Royal Society. Newport Pagnell: The Royal Society, Chicheley Hall; 2019 https:// royalsociety.org/science-events-and-lectures/2019/09/pearls-of-wisdom/.
52. Clamp M, Fry B, Kamal M, Xie X, Cuff J, Lin MF, Kellis M, Lindblad-Toh K, Lander ES. Distinguishing protein-coding and noncoding genes in the human genome. Proc Natl Acad Sci U S A. 2007;104(49):19428-33.

53. Marin R, Cortez D, Lamanna F, Pradeepa MM, Leushkin E, Julien P, Liechti A, Halbert J, Brüning T, Mössinger $K$, et al. Convergent origination of a Drosophila-like dosage compensation mechanism in a reptile lineage. Genome Res. 2017:27(12):1974-87.

54. Modzelewska K, Boer Elena F, Mosbruger Timothy L, Picard D, Anderson D, Miles Rodney R, Kroll M, Oslund W, Pysher Theodore J, Schiffman Joshua D, et al. MEK inhibitors reverse growth of Embryonal brain tumors derived from Oligoneural precursor cells. Cell Rep. 2016;17(5):1255-64.

55. Kaletsky R, Lakhina V, Arey R, Williams A, Landis J, Ashraf J, Murphy CT. The C. elegans adult neuronal IIS/FOXO transcriptome reveals adult phenotype regulators. Nature. 2016;529(7584):92-6.

56. Straub V, Staras K, Kemenes G, Benjamin PR. Endogenous and network properties of Lymnaea feeding central pattern generator interneurons. J Neurophysiol. 2002;88(4):1569-83.

57. Massobrio P, Tessadori J, Chiappalone M, Ghirardi M. In vitro studies of neuronal networks and synaptic plasticity in invertebrates and in mammals using multielectrode arrays. Neural Plasticity. 2015;2015:1-18.

58. Dong N, Senzel A, Li K, Lu TZ, Guo CH, Aleksic M, Feng ZP. MEN1 tumor suppressor gene is required for long-term memory formation in an aversive operant conditioning model of Lymnaea stagnalis. Neuroscience. 2018;379:22-31.

59. Winlow W, Syed NI. The respiratory central pattern generator of Lymnaea. Acta Biol Hung. 1992;43:399-408.

60. Silverman-Gavrila LB, Lu TZ, Prashad RC, Nejatbakhsh N, Charlton MP, Feng ZP. Neural phosphoproteomics of a chronic hypoxia model-Lymnaea stagnalis. Neuroscience. 2009;161(2):621-34.

61. Rosenegger D, Lukowiak K. The participation of NMDA receptors, PKC, and MAPK in the formation of memory following operant conditioning in Lymnaea. Mol Brain. 2010;3(1):1-10.

62. Lu TZ, Kostelecki W, Sun CLF, Dong N, Pérez Velázquez JL, Feng ZP. High sensitivity of spontaneous spike frequency to sodium leak current in a Lymnaea pacemaker neuron. Eur J Neurosci. 2016;44(12):3011-22.

63. Dong N, Feng ZP. Inverse relationship between basal pacemaker neuron activity and aversive long-term memory formation in Lymnaea stagnalis. Front Cell Neurosci. 2017;10(297):297.

64. Adema CM, Van Deutekom-Mulder EC, Van Der Knaap WPW, Sminia T. Schistosomicidal activities of Lymnaea stagnalis haemocytes: the role of oxygen radicals. Parasitology. 1994;109(04):479.

65. Cho H, Davis J, Li X, Smith KS, Battle A, Montgomery SB. High-resolution Transcriptome analysis with long-read RNA sequencing. PLoS One. 2014 9(9):e108095.

66. Nakasugi K, Crowhurst R, Bally J, Waterhouse P. Combining Transcriptome assemblies from multiple de novo assemblers in the Allo-Tetraploid plant Nicotiana benthamiana. PLoS One. 2014:9(3):e91776.

67. Venturini L, Caim S, Kaithakottil GG, Mapleson DL, Swarbreck D. Leveraging multiple transcriptome assembly methods for improved gene structure annotation. GigaScience. 2018;7(8):giy093.

68. Jiménez CR, Spijker S, De Schipper S, Lodder JC, Janse CK, Geraerts WPM, Van Minnen J, Syed NI, Burlingame AL, Smit AB, et al. Peptidomics of a single identified neuron reveals diversity of multiple neuropeptides with convergent actions on cellular excitability. J Neurosci. 2006;26(2):518-29.

69. Brezden BL, Yeoman MS, Gardner DR, Benjamin PR. FMRFamide-Activated $\mathrm{Ca}^{2+}$ Channels in Lymnaea Heart Cells Are Modulated by "SEEPLY," a Neuropeptide Encoded on the Same Gene. J Neurophysiol. 1999;81(4): 1818-26.

70. Zhang G, Vilim FS, Liu D-D, Romanova EV, Yu K, Yuan W-D, Xiao H, Hummon $A B$, Chen T-T, Alexeeva $V$, et al. Discovery of leucokinin-like neuropeptides that modulate a specific parameter of feeding motor programs in the molluscan model, Aplysia. J Biol Chem. 2017;292(46):18775-89.

71. Joris $\mathrm{M}$, Koene JM. Neuro-endocrine control of reproduction in hermaphroditic freshwater snails: mechanisms and evolution. Front Behav Neurosci. 2010;4:167

72. Morishita F, Furukawa Y, Matsushima O, Minakata H. Regulatory actions of neuropeptides and peptide hormones on the reproduction of molluscsThe present review is one of a series of occasional review articles that have been invited by the editors and will feature the broad range of disciplines and expertise represented in our editorial advisory board. Can J Zool. 2010; 88(9):825-45. 
73. Senatore A, Edirisinghe N, Katz PS. Deep mRNA sequencing of the Tritonia diomedea brain transcriptome provides access to gene homologues for neuronal excitability, synaptic transmission and peptidergic signalling. PLoS One. 2015;10(2):e0118321.

74. Mansour TA, Habib MR, Rodríguez LCV, Vázquez AH, Alers JM, Ghezzi A, Croll RP, Brown CT, Miller MW. Central nervous system transcriptome of Biomphalaria alexandrina, an intermediate host for schistosomiasis. BMC Res Notes. 2017;10(1):729.

75. Lu TZ, Feng Z-P. A sodium leak current regulates pacemaker activity of adult central pattern generator neurons in Lymnaea Stagnalis. PLoS One. 2011;6(4):e18745.

76. Dawson TF, Boone AN, Senatore A, Piticaru J, Thiyagalingam S, Jackson D, Davison A, Spafford JD. Gene splicing of an invertebrate beta subunit $(\mathrm{LCav} \beta)$ in the N-terminal and HOOK domains and its regulation of LCav1 and LCav2 calcium channels. PLoS One. 2014;9(4):e92941.

77. Dong N, Lee DWK, Sun H-s, Feng Z-P. Dopamine-mediated calcium channel regulation in synaptic suppression in L. stagnalis interneurons. Channels. 2018;12(1):153-73.

78. Gleichmann M, Mattson MP. Neuronal calcium homeostasis and Dysregulation. Antioxid Redox Signal. 2011;14(7):1261-73.

79. Lu B, Su Y, Das S, Liu J, Xia J, Ren D. The Neuronal Channel NALCN contributes resting sodium permeability and is required for Normal respiratory rhythm. Cell. 2007;129(2):371-83.

80. Moose DL, Haase SJ, Aldrich BT, Lear BC. The narrow abdomen Ion Channel complex is highly stable and persists from development into adult stages to promote behavioral rhythmicity. Front Cell Neurosci. 2017;11:159.

81. Xie L, Gao S, Alcaire Salvador M, Aoyagi K, Wang Y, Griffin Jennifer K, Stagljar I, Nagamatsu S, Zhen M. NLF-1 delivers a sodium Leak Channel to regulate neuronal excitability and modulate rhythmic locomotion. Neuron. 2013; 77(6):1069-82.

82. Himmel NJ, Cox DN. Transient receptor potential channels: current perspectives on evolution, structure, function and nomenclature. Proc R Soc B Biol Sci. 2020;287(1933):20201309.

83. Baylis HA, Goyal K. TRPM channel function in Caenorhabditis elegans. Biochem Soc Trans. 2007;35(1):129-32.

84. Koike C, Numata T, Ueda H, Mori Y, Furukawa T. TRPM1: a vertebrate TRP channel responsible for retinal ON bipolar function. Cell Calcium. 2010;48(23):95-101.

85. Jiao Y, Cao Y, Zheng Z, Liu M, Guo X. Massive expansion and diversity of nicotinic acetylcholine receptors in lophotrochozoans. BMC Genomics. 2019; 20(1):937.

86. Van Nierop P, Bertrand S, Munno DW, Gouwenberg Y, Van Minnen J, Spafford JD, Syed NI, Bertrand D, Smit AB. Identification and functional expression of a family of nicotinic acetylcholine receptor subunits in the central nervous system of the mollusc Lymnaea stagnalis. J Biol Chem. 2006; 281(3):1680-91.

87. Rolls MM, Jegla TJ. Neuronal polarity: an evolutionary perspective. J Exp Biol. 2015;218(4):572-80.

88. Seebacher F. The evolution of metabolic regulation in animals. Comp Biochem Physiol B: Biochem Mol Biol. 2018;224:195-203.

89. Fadda M, Hasakiogullari I, Temmerman L, Beets I, Zels S, Schoofs L. Regulation of feeding and metabolism by neuropeptide $F$ and short neuropeptide $\mathrm{F}$ in invertebrates. Front Endocrinol (Lausanne). 2019;10:64.

90. Altenhoff AM, Studer RA, Robinson-Rechavi M, Dessimoz C. Resolving the Ortholog conjecture: Orthologs tend to be weakly, but significantly, more similar in function than Paralogs. PLoS Comput Biol. 2012;8(5):e1002514.

91. Chen X, Zhang J. The Ortholog conjecture is untestable by the current gene ontology but is supported by RNA sequencing data. PLoS Comput Biol. 2012;8(11):e1002784

92. Rogozin IB, Managadze D, Shabalina SA, Koonin EV. Gene family level comparative analysis of gene expression in mammals validates the Ortholog conjecture. Genome Biol Evol. 2014;6(4):754-62.

93. Kasahara M, Suzuki T, Pasquier LD. On the origins of the adaptive immune system: novel insights from invertebrates and cold-blooded vertebrates. Trends Immunol. 2004;25(2):105-11.

94. Hughes AL. Protein phylogenies provide evidence of a radical discontinuity between arthropod and vertebrate immune systems. Immunogenetics. 1998:47(4):283-96.

95. Bosch M, Hayashi Y. Structural plasticity of dendritic spines. Curr Opin Neurobiol. 2012;22(3):383-8.
96. Fredriksson R, Schiöth HB. The repertoire of G-protein-coupled receptors in fully sequenced genomes. Mol Pharmacol. 2005;67(5):1414-25.

97. Krishnan A, Mustafa A, Almén MS, Fredriksson R, Williams MJ, Schiöth HB. Evolutionary hierarchy of vertebrate-like heterotrimeric $\mathrm{G}$ protein families. Mol Phylogenet Evol. 2015;91:27-40.

98. Winlow W, Benjamin PR. Neuronal mapping of the brain of the pond snail, Lymnaea stagnalis (L.). In: Salanki J, editor. Neurobiology of Invertebrates, Gastropoda Brain, vol. 41. Budapest: Akademiai Kiado; 1976. p. 59.

99. Hui K, Fei GH, Saab BJ, Su J, Roder JC, Feng ZP. Neuronal calcium sensor-1 modulation of optimal calcium level for neurite outgrowth. Development. 2007;134(24):4479-89.

100. Natsidis P, Schiffer PH, Salvador-Martínez I, Telford MJ. Computational discovery of hidden breaks in $28 \mathrm{~S}$ ribosomal RNAs across eukaryotes and consequences for RNA Integrity Numbers. Sci Rep. 2019;9(1):19477.

101. Alberti A, Poulain J, Engelen S, Labadie K, Romac S, Ferrera I, Albini G, Aury J-M, Belser C, Bertrand A, et al. Viral to metazoan marine plankton nucleotide sequences from the Tara oceans expedition. Scientific Data. 2017;4(1):170093.

102. Kopylova E, Noé L, Touzet H. SortMeRNA: fast and accurate filtering of ribosomal RNAs in metatranscriptomic data. Bioinformatics. 2012;28(24):3211-7.

103. Andrews S. FastQC: a quality control tool for high throughput sequence data, vol. 1; 2010. p. 1-1.

104. Song L, Florea L. Rcorrector: efficient and accurate error correction for Illumina RNA-seq reads. GigaScience. 2015;4(1):1-8.

105. Dobin A, Davis CA, Schlesinger F, Drenkow J, Zaleski C, Jha S, Batut P, Chaisson M, Gingeras TR. STAR: ultrafast universal RNA-seq aligner. Bioinformatics. 2013;29(1):15-21.

106. Pertea M, Pertea GM, Antonescu CM, Chang T-C, Mendell JT, Salzberg SL. StringTie enables improved reconstruction of a transcriptome from RNA-seq reads. Nat Biotechnol. 2015:33(3):290-5.

107. Shao M, Kingsford C. Accurate assembly of transcripts through phasepreserving graph decomposition. Nat Biotechnol. 2017;35(12):1167-9.

108. Liu R, Dickerson J. Strawberry: fast and accurate genome-guided transcript reconstruction and quantification from RNA-Seq. PLoS Comput Biol. 2017; 13(11):e1005851.

109. Grabherr MG, Haas BJ, Yassour M, Levin JZ, Thompson DA, Amit I, Adiconis X Fan L, Raychowdhury R, Zeng Q, et al. Full-length transcriptome assembly from RNA-Seq data without a reference genome. Nat Biotechnol. 2011;29(7):644-52.

110. Gilbert D. EvidentialGene: mRNA Transcript Assembly Software; 2013.

111. Visser EA, Wegrzyn JL, Steenkmap ET, Myburg AA, Naidoo S. Combined de novo and genome guided assembly and annotation of the Pinus patula juvenile shoot transcriptome. BMC Genomics. 2015;16(1):1057.

112. Mamrot J, Legaie R, Ellery SJ, Wilson T, Seemann T, Powell DR, Gardner DK, Walker DW, Temple-Smith P, Papenfuss AT, et al. De novo transcriptome assembly for the spiny mouse (Acomys cahirinus). Sci Rep. 2017;7(1):8996.

113. Patro R, Duggal G, Love MI, Irizarry RA, Kingsford C. Salmon provides fast and bias-aware quantification of transcript expression. Nat Methods. 2017; 14(4):417-9.

114. Simão FA, Waterhouse RM, loannidis P, Kriventseva EV, Zdobnov EM. BUSCO: assessing genome assembly and annotation completeness with single-copy orthologs. Bioinformatics. 2015;31(19):3210-2.

115. Fu L, Niu B, Zhu Z, Wu S, Li W. CD-HIT: accelerated for clustering the nextgeneration sequencing data. Bioinformatics. 2012;28(23):3150-2.

116. Chen S, Zhou Y, Chen Y, Gu J. fastp: an ultra-fast all-in-one FASTQ preprocessor. Bioinformatics. 2018;34(17):i884-90.

117. MacManes MD, Eisen MB. Characterization of the transcriptome, nucleotide sequence polymorphism, and natural selection in the desert adapted mouse Peromyscus eremicus. PeerJ. 2014;2:e642.

118. Emms DM, Kelly S. OrthoFinder: solving fundamental biases in whole genome comparisons dramatically improves orthogroup inference accuracy. Genome Biol. 2015;16(1):157.

119. Conway JR, Lex A, Gehlenborg N. UpSetR: an R package for the visualization of intersecting sets and their properties. Bioinformatics. 2017;33(18):2938-40.

120. Durinck S, Spellman PT, Birney E, Huber W. Mapping identifiers for the integration of genomic datasets with the R/bioconductor package biomaRt. Nat Protoc. 2009;4(8):1184-91.

121. Reimand J, Arak T, Adler P, Kolberg L, Reisberg S, Peterson H, Vilo J. g: Profiler-a web server for functional interpretation of gene lists (2016 update). Nucleic Acids Res. 2016;44(W1):W83-9. 
122. Supek F, Bošnjak M, Škunca N, Šmuc T. REVIGO summarizes and visualizes long lists of gene ontology terms. PLoS One. 2011;6(7):e21800.

123. Notredame C, Higgins DG, Heringa J. T-coffee: a novel method for fast and accurate multiple sequence alignment. J Mol Biol. 2000;302(1):205-17.

124. Capella-Gutierrez S, Silla-Martinez JM, Gabaldon T. trimAl: a tool for automated alignment trimming in large-scale phylogenetic analyses. Bioinformatics. 2009;25(15):1972-3.

125. Jones DT, Taylor WR, Thornton JM. The rapid generation of mutation data matrices from protein sequences. Bioinformatics. 1992;8(3):275-82.

126. Kumar S, Stecher G, Li M, Knyaz C, Tamura K. MEGA X: molecular evolutionary genetics analysis across computing platforms. Mol Biol Evol. 2018;35(6):1547-9.

127. Yu G, Smith DK, Zhu H, Guan Y, Lam TTY. ggtree: an R package for visualization and annotation of phylogenetic trees with their covariates and other associated data. Methods Ecol Evol. 2016;8(1): 28-36.

128. Fernandez NF, Gundersen GW, Rahman A, Grimes ML, Rikova K, Hornbeck P, Ma'ayan A. Clustergrammer, a web-based heatmap visualization and analysis tool for high-dimensional biological data. Scientific Data. 2017;4(1): 170151

\section{Publisher's Note}

Springer Nature remains neutral with regard to jurisdictional claims in published maps and institutional affiliations.

Ready to submit your research? Choose BMC and benefit from:

- fast, convenient online submission

- thorough peer review by experienced researchers in your field

- rapid publication on acceptance

- support for research data, including large and complex data types

- gold Open Access which fosters wider collaboration and increased citations

- maximum visibility for your research: over $100 \mathrm{M}$ website views per year

At BMC, research is always in progress.

Learn more biomedcentral.com/submissions 